\title{
UNIQUENESS IN BOUNDED MOMENT PROBLEMS
}

\author{
HANS G. KELLERER
}

\begin{abstract}
Let $(X, \mathfrak{A}, \mu)$ be a $\sigma$-finite measure space and $\mathscr{K}$ be a linear subspace of $\mathscr{L}_{1}(\mu)$ with $\operatorname{supp} \mathscr{K}=X$. The following inverse problem is treated: Which sets $A \in \mathfrak{A}$ are " $\mathscr{K}$-determined" within the class of all functions $g \in \mathscr{L}_{\infty}(\mu)$ satisfying $0 \leq g \leq 1$, i.e. when is $g=1_{A}$ the unique solution of $\int f g d \mu=\int f 1_{A} d \mu, f \in \mathscr{K}$ ? Recent results of Fishburn et al. and Kemperman show that the condition $A=\{f \geq 0\}$ for some $f \in \mathscr{K}$ is sufficient but not necessary for uniqueness. To obtain a complete characterization of all $\mathscr{K}$-determined sets, $\mathscr{K}$ has to be enlarged to some hull $\mathscr{K}^{*}$ by extending the usual weak convergence to limits not in $\mathscr{L}_{1}(\mu)$. Then one of the main results states that $A$ is $\mathscr{K}$-determined if and only if there is a representation $A=\left\{f^{*}>0\right\}$ and $X \backslash A=\left\{f^{*}<0\right\}$ for some $f^{*} \in \mathscr{K}^{*}$.
\end{abstract}

\section{INTRODUCTION}

It is an immediate consequence of the Fourier inversion formula that a finite mass distribution $\mu_{0}$ on $\mathbb{R}^{n}$ is uniquely determined by all its one-dimensional projections, i.e. by the image measures $\varphi\left(\mu_{0}\right)$ with respect to all linear maps $\varphi: \mathbb{R}^{n} \rightarrow \mathbb{R}$. In applications as in tomography, however, only a finite number of these projections can be observed. For simplicity let $\mu_{0}$ be absolutely continuous with respect to Lebesgue measure; then it turns out that-except for the trivial case $\mu_{0}=0$-a reconstruction is never possible (see $\S 6$ ). In most applications, however, some upper bound $\mu$ for $\mu_{0}$ is available. Thus, given projections $\varphi_{1}, \ldots, \varphi_{k}$, the following "bounded moment problem" arises: Which measures $\mu_{0}$ are uniquely determined by their images $\varphi_{i}\left(\mu_{0}\right)$ under the side condition $\mu_{0} \leq \mu$ ? Since the set of measures meeting these constraints is convex, it is no surprise that a necessary condition requires $\mu_{0}$ to be a restriction of $\mu$ to some subset $A$ (see $\S 6$ ). Translated via the Radon-Nikodym theorem from measures to functions the problem takes the following form: The indicator functions of which sets $A \subset \mathbb{R}^{n}$ are-up to null sets-uniquely determined, within the class of all functions $g$ on $\mathbb{R}^{n}$ satisfying $0 \leq g \leq 1$, by the integrals $\int_{X} f \circ \varphi_{i} 1_{A} d \mu, 1 \leq i \leq k$, for all bounded functions $f$ on $\mathbb{R}$ ?

Kuba and Volcic [12] and Fishburn et al. [3, 4] seem to be the first who studied this problem, specializing it to the classical "marginal" situation, i.e. the case where $\mu$ is (a restriction of) Lebesgue measure and the canonical projections $\pi_{1}, \ldots, \pi_{n}$ play the role of the maps $\varphi_{1}, \ldots, \varphi_{k}$. In [12] the authors restrict the study to dimension 2 and, making essential use of a result due to Lorentz

Received by the editors December 21, 1990.

1980 Mathematics Subject Classification (1985 Revision). Primary 44A60; Secondary 28A35, 46G10, 52A07. 
[15], give three different characterizations of the sets in question. In [3] it is shown that, without dimension restrictions, a representation

$$
A=\left\{x \in \mathbb{R}^{n}: f_{1}\left(x_{1}\right)+\cdots+f_{n}\left(x_{n}\right) \geq 0\right\}
$$

with appropriate functions $f_{i}$ is a sufficient condition for uniqueness. In a preliminary version the authors conjectured this special structure of $A$ (called "additivity") to be necessary, too. This, however, was disproved by Kemperman [9], who supplied a counterexample in dimension 3. In [8] he generalized the problem by considering an arbitrary measure space $(X, \mathfrak{A}, \mu)$ and replacing the functions $f \circ \pi_{i}$ by a linear space $\mathscr{K}$ of integrable functions-as was done independently by the present author. The problem then reads: For which sets $A \in \mathfrak{A}$ do the equations

$$
\int_{A} f d \mu=\int_{X} f g d \mu \text { for } f \in \mathscr{K}
$$

under the additional assumption $0 \leq g \leq 1$ imply $g=1_{A}$ modulo $\mu$ ?

It is the main aim of this paper to derive conditions for uniqueness of $A$ in the above sense that are not only sufficient but also necessary. As it turns out, this amounts to a careful extension of $\mathscr{K}$ to a "hull" $\mathscr{K}^{*}$ such that a representation

$$
A=\left\{f^{*}>0\right\} \text { and } X \backslash A=\left\{f^{*}<0\right\}
$$

for some $f^{*} \in \mathscr{K}^{*}$ is equivalent to uniqueness. Partly due to a somewhat different approach, there is only a minor overlap with [8,9] as will become clear from the following survey of the subsequent sections.

Since the class of " $\mathscr{K}$-determined" sets as introduced in Definition (1.2) turns out to be stable with respect to complementation (see (1.5)), a fundamental device is the limitation to notions that are symmetric in $A$ and its complement $X \backslash A$. This suggests in particular the study of " $\mathscr{K}$-separated" sets as introduced in Definition (1.6), which are contained in the class of $\mathscr{K}$-determined sets (see (1.7)). This subclass, however, can be very modest unless $\mathscr{K}$ is assumed to be closed, as is shown by Example (1.8). Another difficulty can be caused by the existence of a nontrivial subset of $X$ where all functions $f \in \mathscr{K}$ vanish and therefore provide no information at all. This motivates the introduction of a "support" of $\mathscr{K}$ in Definition (1.9) and explains the importance of Lemma (1.10). One consequence is Proposition (1.11) stating that a full support of $\mathscr{K}$ is not only necessary but also sufficient for the existence of $\mathscr{K}$-determined sets. The section concludes with a geometric characterization of these sets as extreme points of a related convex set (see (1.14)).

Interpreting in $\S 2$ the reconstruction of a set $A$ from the associated integrals $\int_{A} f d \mu, f \in \mathscr{K}$, as an extension of some linear functional, it is natural to employ the Hahn-Banach theorem. This provides in Theorem (2.1) a central criterion for uniqueness. To derive from it an explicit representation of $\mathscr{K}$ determined sets, it is crucial to extend the notion of weak convergence from integrable functions to a larger class. This leads to the definition of a hull $\mathscr{K}^{*}$ of $\mathscr{K}$ (see (2.4)) and allows a complete characterization of $\mathscr{K}$-determined sets in Theorem (2.5), representing $A$ and $X \backslash A$ by means of a function $f^{*} \in \mathscr{K}^{*}$ in the above sense.

Since the passage from $\mathscr{K}$ to $\mathscr{K}^{*}$ is a delicate limiting process, it is of interest to find a more constructive access to as many $\mathscr{K}$-determined sets as 
possible. To this end, in $\S 3$ the following arguments will be combined: (1) the $\mathscr{K}$-separated sets result from maximizing linear functionals associated with the functions $f \in \mathscr{K},(2)$ the $\mathscr{K}$-determined sets may be interpreted as extreme points of some convex set, (3) in the finite-dimensional case these points can be found by maximizing a finite number of linear functionals recursively. Without dimension restriction but within the framework of measure theory this suggests the use of sequences $f_{n} \in \mathscr{K}, n \in \mathbb{N}$, in the present context. As will follow from Example (3.3), however, it is necessary to work with countable ordinals instead of ordinary sequences. This leads in Definition (3.2) to sets that are " $\mathscr{K}$-separated of order $\gamma$," which have their origin in the special case $\gamma=1$. As will be seen, these classes of sets, which for finite $\gamma$ are also considered by Kemperman [8, 9], may increase strictly for increasing parameter $\gamma$. By means of the criterion (2.5) they can be shown to consist indeed of $\mathscr{K}$-determined sets (see (3.4)). As is demonstrated, however, by Example (3.5), even on a countable space $X$ their union does not necessarily exhaust the class of all $\mathscr{K}$-determined sets. An exception is provided by the case of a finite-dimensional space $\mathscr{K}$, as follows easily from the arguments (1)-(3) above (see (3.8)) and is proved in [8] by ad hoc methods.

In view of the gap between $\mathscr{K}$-separated sets and $\mathscr{K}$-determined sets it is a natural question whether at least an approximation theorem is available. After the introduction of an appropriate topology in the $\sigma$-algebra $\mathfrak{A}, \S 4$ first investigates whether the class of all $\mathscr{K}$-determined sets itself is closed with respect to it. This turns out to be true in the finite-dimensional case (see (4.1)), while Example (4.2) shows to what extent this can fail otherwise. Therefore the notion of "almost $\mathscr{K}$-determined" sets is introduced in Definition (4.4) and their connection with $\mathscr{K}$-separated sets is clarified in Theorem (4.6). By means of this criterion the representation (2.5) can be carried over to almost $\mathscr{K}$-determined sets, replacing the hull $\mathscr{K}^{*}$ by the set $\mathscr{K}^{* *}$ of all pointwise limits of sequences in $\mathscr{K}$ (see (4.8)). Moreover, Theorem (4.9) solves the approximation problem mentioned at the beginning of this paragraph.

The following section considers a weaker notion of uniqueness, which is more adequate for some applications but less convenient to work with. The point in Definition (5.1) is to compare a set $A$ only with indicator functions instead of all functions $g$ satisfying $0 \leq g \leq 1$. It is typical, however, for applications that the subspace $\mathscr{K}$ is not too large, i.e. "thin" in the sense of Definition (5.3), and in this case both notions coincide (see (5.4)).

The concluding section is devoted to the classical geometric situation as explained at the beginning of this introduction. From results proved by the author [7] long ago it follows that strong and weak uniqueness agree in this case. As it turns out, the results concerning dimension $n=2$ resp. $n>2$ differ completely. Using results in [3] and [12] in the first case, Proposition (6.2) proves the coincidence of $\mathscr{K}$-separated and almost $\mathscr{K}$-determined sets, including thus all other types. In contrast, in dimension $n>2$ there are counterexamples (1) due to Kemperman [9] showing that the order of separation cannot be dispensed with in general and (2) by Fishburn et al. [3] showing that the class of $\mathscr{K}$-determined sets fails to be closed even with respect to monotone sequences. Thus it remains to find a counterexample (3) demonstrating that the classes of generalized $\mathscr{K}$-separated sets again need not exhaust the class of all $\mathscr{K}$ determined sets. This is done in Proposition (6.4), supplying suitable sets of a very simple structure. 
Finally, it should be pointed out that all proper geometric aspects, as provided for instance by convexity, are disregarded in this paper. For related results see the survey paper by Gardner [5] and the references therein.

\section{Notation}

Given a (nonnegative) measure space $(X, \mathfrak{A}, \mu)$, the function spaces $\mathscr{L}_{1}(\mu)$ and $\mathscr{L}_{\infty}(\mu)$ have their usual meaning. Since the norm in $\mathscr{L}_{\infty}(\mu)$ will play no role, the norm in $\mathscr{L}_{1}(\mu)$ is simply denoted by $\|\cdot\|$.

$\mathscr{L}_{0}(\mu)$ resp. $\widetilde{\mathscr{L}}_{0}(\mu)$ is written for the space of measurable functions with values in $\mathbb{R}$ resp. $\widetilde{\mathbb{R}}$, the extended real line. Here, as above, functions that agree modulo $\mu$ are identified and the same convention holds for sets in the $\sigma$-algebra.

Since suppressing $\mu$ in equations and inequalities may, however, be misleading in statements like $A=\varnothing$, as a rule the symbols $\underset{\mu}{=}$ and $\underset{\mu}{\leq}$ resp. $\underset{\mu}{\subset}$ are used in the sequel.

For $f \in \overline{\mathscr{L}}_{0}(\mu)$ and $A \in \mathfrak{A}$ the $\mu$-essential supremum of $f$ on $A$ is denoted by $\mu$-sup $\{f(x): x \in A\}$; for a family of functions $f_{i} \in \widetilde{\mathscr{L}}_{0}(\mu)$, resp. sets $A_{i} \in \mathfrak{A}, i \in I$, the $\mu$-essential supremum, if defined, is denoted by $\mu$-sup $\operatorname{sel}_{i \in I} f_{i}$, resp. $\mu$ - $\sup _{i \in I} A_{i}$ (infima will not occur).

Finally, for the $\sigma$-algebra generated by any family $\mathscr{F}=\left\{f_{i}: i \in I\right\}$ of real-valued functions on $X$ the notations $\sigma(\mathscr{F})$ as well as $\sigma\left(f_{i}, i \in I\right)$ are used.

\section{BASIC FACTS}

Throughout this paper the following conventions hold:

(a) $(X, \mathfrak{A}, \mu)$ is a $\sigma$-finite measure space,

(b) $\mathscr{K}$ is a linear subspace of $\mathscr{L}_{1}(\mu)$.

Concerning (a), it should be mentioned that some of the subsequent results extend easily to a localizable measure space. On the other hand, the $\sigma$-finite case formally can be reduced to the finite one, multiplying the measure $\mu$ by a strictly positive function $h \in \mathscr{L}_{1}(\mu)$ and all functions in $\mathscr{K}$ by $1 / h$-at the expense, however, of obscuring some essential points. Concerning (b), the linearity assumption is obviously no real restriction, because, given the integrals $\int_{X} f_{i} g d \mu$ of a function $g \in \mathscr{L}_{\infty}(\mu)$ for some functions $f_{i} \in \mathscr{L}_{1}(\mu)$, these integrals are known for all their linear combinations. By a similar argument $\mathscr{K}$ could be replaced by its closure $\overline{\mathscr{K}}$ as well, but this would unnecessarily weaken some of the subsequent results.

The subspace $\mathscr{K}$ induces the following equivalence relation:

$$
\begin{aligned}
& \text { Definition. Two functions } g_{i} \in \mathscr{L}_{\infty}(\mu) \text { fulfil } g_{1} \underset{\mathscr{K}}{{ }^{\prime}} g_{2} \text {, if } \\
& \qquad \int_{X} f g_{1} d \mu=\int_{X} f g_{2} d \mu \text { for all } f \in \mathscr{K} .
\end{aligned}
$$

This equivalence relation yields the quotient space of $\mathscr{L}_{\infty}(\mu)$ with respect to the closed linear subspace

$$
\mathscr{K}^{\perp}=\left\{g \in \mathscr{L}_{\infty}(\mu): \int_{X} f g d \mu=0 \text { for all } f \in \mathscr{K}\right\} .
$$


Therefore the relation $\widetilde{\mathscr{K}}$ is compatible not only with equality modulo $\mu$ but also with the linear structure in $\mathscr{L}_{\infty}(\mu)$. In a natural way $(1.1)$ defines an equivalence relation in the $\sigma$-algebra $\mathfrak{A}$, denoted again by $\widetilde{\mathscr{K}}$, which, however, need not be compatible with the lattice structure in $\mathfrak{A}$.

The main object of this paper is the study of the following class of sets:

(1.2) Definition. With the notation

$$
\mathscr{G}=\left\{g \in \mathscr{L}_{\infty}(\mu): 0 \underset{\mu}{\leq} \underset{\mu}{\leq} 1\right\}
$$

the class $\mathfrak{D}(\mathscr{K})$ consists of all " $\mathscr{K}$-determined" sets $A \in \mathfrak{A}$, satisfying the condition

$$
1_{A} \underset{\mathscr{K}}{\sim} g \in \mathscr{G} \text { implies } g=1_{\mu}
$$

This class is clearly invariant under the passage from $\mathscr{K}$ to $\overline{\mathscr{K}}$. Moreover, it increases when $\mathscr{K}$ increases, the extreme cases being given by

$$
\mathfrak{D}(\mathscr{K})=\varnothing \text { for } \mathscr{K}=\{0\} \quad \text { and } \mathfrak{D}(\mathscr{K})=\mathfrak{A} \text { for } \mathscr{K}=\mathscr{L}_{1}(\mu) ;
$$

here $\mu \neq 0$ has to be assumed in the first equation.

Equally simple is the following case:

(1.3) Example. Assume $0<\mu(X)<\infty$ and let $\mathscr{K}$ consist of all constant functions. Then

$$
1_{A} \underset{\mathscr{K}}{\sim}(\mu(A) / \mu(X)) 1_{X} \in \mathscr{G} \text { for all } A \in \mathfrak{A}
$$

thus $\mathfrak{D}(\mathscr{K})$ contains only the extreme sets $\varnothing$ and $X$.

As defined in (1.2) the class $\mathfrak{D}(\mathscr{K})$ depends on the underlying $\sigma$-algebra, but this dependence is only formal:

(1.4) Proposition. Let the measure $\mu$ be $\sigma$-finite on $\mathfrak{A}_{0}=\sigma(\mathscr{K})$. If $\mathfrak{D}_{0}(\mathscr{K})$ denotes the analogue of $\mathfrak{D}(\mathscr{K})$ with $\mathfrak{A}_{0}$ replacing $\mathfrak{A}$, then

$$
A \in \mathfrak{D}(\mathscr{K}) \text { if and only if } A=A_{0} \in \mathfrak{D}_{0}(\mathscr{K}) \text {. }
$$

Proof. 0. If $\mathscr{G}_{0}$ denotes similarly the analogue of $\mathscr{G}$, the Radon-Nikodym theorem assigns to each $g \in \mathscr{G}$ a function $g_{0} \in \mathscr{G}_{0}$ such that

$$
\int_{B} g d \mu=\int_{B} g_{0} d \mu \text { for all } B \in \mathfrak{A}_{0},
$$

hence in particular

$$
\int_{X} f g d \mu=\int_{X} f g_{0} d \mu \text { for all } f \in \mathscr{K} .
$$

1. Assume now $A \in \mathfrak{D}(\mathscr{K})$ and consider the function $g_{0}=\left(1_{A}\right)_{0}$ according to part 0 of the proof. Then by (2)

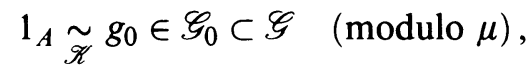

hence $g_{0}=1_{A}$ by uniqueness and thus $g_{0} \underset{\mu}{=} 1_{A_{0}}$ for some $A_{0} \in \mathfrak{A}_{0}$. But this implies first $A_{0}=\underset{\mu}{=}$ and then $A_{0} \in \mathfrak{D}_{0}(\mathscr{K})$ due to $A \in \mathfrak{D}(\mathscr{K})$. 
2. Conversely, assume $A \underset{\mu}{\bar{\mu}} A_{0} \in \mathfrak{D}_{0}(\mathscr{K})$ and consider an equivalence $1_{A} \underset{\mathscr{K}}{\sim} g$ with $g \in \mathscr{G}$. Then by (2) $1_{A_{0}} \underset{\mu}{=} 1_{A} \underset{\mathscr{H}}{\widetilde{g}} g_{0} \in \mathscr{G}_{0}$, hence $g_{0} \underset{\mu}{=} 1_{A_{0}}$ by uniqueness and thus by (1)

$$
\int_{B} g d \mu=\int_{B} g_{0} d \mu=\int_{B} 1_{A_{0}} d \mu \text { for all } B \in \mathfrak{A}_{0} .
$$

By choosing $B=A_{0}$ resp. $X \backslash A_{0}$ this implies $g_{\mu}^{=} 1_{A_{0}}$ due to $0 \leq \underset{\mu}{\leq} g_{\mu} 1$ and therefore indeed $g={ }_{\mu}{ }_{A}$.

Clearly, this result contains the preceding example as a special case. Moreover, assuming $\mu$ to be $\sigma$-finite on $\mathfrak{A}_{0}$ is no real restriction, because this condition holds whenever $\mathfrak{D}(\mathscr{K}) \neq \varnothing$, as will follow from (1.10) and (1.11).

Concerning stability properties, it will be seen in the sequel that the class $\mathfrak{D}(\mathscr{K})$ in general enjoys only the following one:

(1.5) Proposition. $\mathfrak{D}(\mathscr{K})$ is stable with respect to complementation.

Proof. If $A \in \mathfrak{D}(\mathscr{K})$, an equivalence

$$
1_{X \backslash A} \tilde{\mathscr{K}} g \in \mathscr{G}
$$

leads to

$$
1_{A} \tilde{\mathscr{K}} 1-g \in \mathscr{G},
$$

hence $1-g_{\mu}^{=} 1_{A}$ or $g_{\mu}^{=} 1_{X \backslash A}$, i.e. $X \backslash A \in \mathfrak{D}(\mathscr{K})$.

This result suggests as a general principle to study only notions that are symmetric in $A$ and its complement. Since $\mathscr{K}$ together with $f$ contains $-f$, this holds in the following case:

(1.6) Definition. The class $\mathfrak{S}(\mathscr{K})$ consists of all " $\mathscr{K}$-separated" sets $A \in \mathfrak{A}$, allowing a representation

$$
A \underset{\mu}{=}\{f>0\} \quad \text { and } \quad X \backslash A \underset{\mu}{=}\{f<0\}
$$

for some function $f \in \mathscr{K}$.

Thus, for instance, the sets $\varnothing$ and $X$ are contained in $\mathfrak{S}(\mathscr{K})$ if and only if $\mathscr{K}$ contains a function $f$ that is strictly positive.

The importance of this class is easily established:

(1.7) Proposition. $\mathfrak{S}(\mathscr{K})$ is a subclass of $\mathfrak{D}(\mathscr{K})$.

Proof. If $A$ is represented by $f$ in the sense of (1.6), $1_{A}$ is uniquely determined as that function $g \in \mathscr{G}$ for which the integral $\int_{X} f g d \mu$ attains its maximal value.

The classes $\mathfrak{S}(\mathscr{K})$ and $\mathfrak{D}(\mathscr{K})$ may differ extremely:

(1.8) Example. Let $(X, \mathfrak{A}, \mu)$ be given by Lebesgue measure on the real line and choose for $\mathscr{K}$ the linear space of all continuous functions with compact support (modulo $\mu$ ). Then $\mathfrak{S}(\mathscr{K})=\varnothing$ and $\mathfrak{D}(\mathscr{K})=\mathfrak{A}$, because $\mu(f=0)=\infty$ for all $f \in \mathscr{K}$, while any function $g \in \mathscr{L}_{\infty}(\mu)$ is clearly determined by the integrals $\int_{X} f g d \mu, f \in \mathscr{K}$. 
The crucial point in this example lies in the fact that the subspace $\mathscr{K}$ is not closed in $\mathscr{L}_{1}(\mu)$. Indeed, its closure equals $\mathscr{L}_{1}(\mu)$, and it is immediate that always

$$
\mathfrak{S}(\mathscr{K})=\mathfrak{A} \text { for } \mathscr{K}=\mathscr{L}_{1}(\mu) .
$$

Therefore, counterexamples concerning $\mathfrak{S}(\mathscr{K})$ are convincing only for closed $\mathscr{K}$.

Another problem may be caused by the existence of a set $A$ of positive measure on which all functions $f \in \mathscr{K}$ vanish. This suggests the following notion, which makes use of the fact that the measure $\mu$ is $\sigma$-finite:

$$
\begin{aligned}
& \text { Definition. The "support" of } \mathscr{K} \text { is given by } \\
& \operatorname{supp} \mathscr{K}=\mu \text {-sup }\{f \neq 0\} .
\end{aligned}
$$

This support is clearly invariant under the passage from $\mathscr{K}$ to $\overline{\mathscr{K}}$. As follows from (1.8), however, assuming $\mathscr{K}$ to be closed is essential for the following result:

$$
\text { Lemma. If } \mathscr{K} \text { is closed, then }
$$

$$
\operatorname{supp} \mathscr{K} \underset{\mu}{=}\left\{f_{0} \neq 0\right\} \quad \text { for some } f_{0} \in \mathscr{K} .
$$

Proof. 1. According to the definition there exist functions $f_{n} \in \mathscr{K}$ with

$$
\sup \mathscr{K} \underset{\mu}{=} \bigcup_{n \in \mathbb{N}}\left\{f_{n} \neq 0\right\},
$$

where convergence of $\sum_{n \in \mathbb{N}}\left\|f_{n}\right\|$ can be achieved by taking suitable multiples. Therefore, after a modification of $f_{n}$ on some null set if necessary, it may be assumed that

$$
\sum_{n \in \mathbb{N}}\left|f_{n}(x)\right|<\infty \text { for all } x \in X .
$$

With the notations

$$
T_{n}=[0,1], \quad T_{\bar{n}}=\prod_{n \neq m \in \mathbb{N}} T_{m} \quad \text { and } \quad T=T_{n} \times T_{\bar{n}} \text { for } n \in \mathbb{N}
$$

this implies that the functions

$$
f_{t}=\sum_{n \in \mathbb{N}} t_{n} f_{n} \quad \text { for } t=\left(t_{n}, n \in \mathbb{N}\right) \in T
$$

are well-defined and belong to the closed subspace $\mathscr{K}$.

2. Consider next the sets

$$
A_{t}^{n}=\left\{f_{n} \neq 0, f_{t}=0\right\} \quad \text { for } n \in \mathbb{N} \text { and } t \in T .
$$

Since the sets $A_{\left(t_{n}, t_{\bar{n}}\right)}^{n}, t_{n} \in T_{n}$, are pairwise disjoint for fixed $t_{\bar{n}} \in T_{\bar{n}}$ and the measure $\mu$ is $\sigma$-finite, the set

$$
S_{t_{\bar{n}}}^{n}=\left\{t_{n} \in T_{n}: \mu\left(A_{\left(t_{n}, t_{\bar{n}}\right)}^{n}\right)>0\right\}
$$

is countable for every $t_{\bar{n}} \in T_{\bar{n}}$. 
3. Denote now by $\nu_{n}$ Lebesgue measure on $T_{n}$ and by $\nu_{\bar{n}}$ and $\nu$ the corresponding product measures on $T_{\bar{n}}$ and $T$, respectively. Then

$$
\nu\left(\left\{t \in T: \mu\left(A_{t}^{n}\right)>0\right\}\right)=\int_{T_{\bar{n}}} \nu_{n}\left(S_{t_{\bar{n}}}^{n}\right) d \nu_{\bar{n}}=0
$$

for all $n \in \mathbb{N}$ by part 2 of the proof. Therefore also

$$
\nu\left(\bigcup_{n \in \mathbb{N}}\left\{t \in T: \mu\left(A_{t}^{n}\right)>0\right\}\right)=0,
$$

which implies the existence of some $t \in T$ such that

$$
\mu\left(A_{t}^{n}\right)=0 \text { for all } n \in \mathbb{N} .
$$

This in turn yields

$$
\bigcup_{n \in \mathbb{N}}\left\{f_{n} \neq 0\right\} \underset{\mu}{\subset}\left\{f_{t} \neq 0\right\},
$$

i.e. $f_{t}$ may be chosen for $f_{0}$.

By means of this lemma the next result is immediate:

(1.11) Proposition. $\mathfrak{D}(\mathscr{K}) \neq \varnothing$ is equivalent to $\operatorname{supp} \mathscr{K} \underset{\mu}{=} X$.

Proof. 1. Since $A_{1} \underset{\mathscr{K}}{\sim} A_{2}$, whenever the symmetric difference of $A_{1}$ and $A_{2}$ is contained in $X \backslash \operatorname{supp} \mathscr{K}$, the condition supp $\mathscr{K} \underset{\mu}{=} X$ is obviously necessary for $\mathfrak{D}(\mathscr{K}) \neq \varnothing$.

2. Conversely, if it is satisfied, there exists by (1.10) a function $f_{0} \in \overline{\mathscr{K}}$ with $\mu\left(f_{0}=0\right)=0$ and thus by $(1.7)$

$$
\left\{f_{0}>0\right\} \in \mathfrak{S}(\overline{\mathscr{K}}) \subset \mathfrak{D}(\overline{\mathscr{K}})=\mathfrak{D}(\mathscr{K}) .
$$

If $\mathfrak{D}(\mathscr{K})$ is nonempty, $\varnothing$ and $X$ may be the only $\mathscr{K}$-determined sets, as is the case in (1.3), where $\operatorname{dim} \mathscr{K}=1$. There are, however, other sets in $\mathfrak{D}(\mathscr{K})$ as soon as $\operatorname{dim} \mathscr{K}>1$. Indeed, by $(1.10)$ choose a function $f_{0} \in \overline{\mathscr{K}}$ with $\mu\left(f_{0}=0\right)=0$ and another function $f_{1} \in \overline{\mathscr{K}}$ such that $f_{0}$ and $f_{1}$ are linearly independent. Then $e=f_{1} / f_{0}$ is not constant modulo $\mu$, hence $\mu(e<t)>0$, $\mu(e=t)=0, \mu(e>t)>0$ for some constant $t$. If $f_{0}$ does not change its sign modulo $\mu$, the function $f=t f_{0}-f_{1} \in \overline{\mathscr{K}}$ yields the nontrivial sets $\{f>0\}$ and $\{f<0\}$ in $\mathfrak{D}(\overline{\mathscr{K}})=\mathfrak{D}(\mathscr{K})$, while otherwise $\left\{f_{0}>0\right\}$ and $\left\{f_{0}<0\right\}$ will do.

On the other hand, it may happen that all sets other than $\varnothing$ and $X$ are $\mathscr{K}$-determined:

(1.12) Example. Assume $0<\mu(X)<\infty$ and consider the closed subspace

$$
\mathscr{K}=\left\{f \in \mathscr{L}_{1}(\mu): \int_{X} f d \mu=0\right\} .
$$

Then obviously $\varnothing \widetilde{\mathscr{H}} X$, while each set $A$ with $0<\mu(A)<\mu(X)$ is even $\mathscr{K}$-separated, as can be seen by choosing the function

$$
f=\mu(X \backslash A) 1_{A}-\mu(A) 1_{X \backslash A} \in \mathscr{K} .
$$


In addition, this example shows that the class $\mathfrak{D}(\mathscr{K})$ as well as $\mathfrak{S}(\mathscr{K})$ need not be stable with respect to monotone sequences.

To derive a first criterion for $\mathscr{K}$-determined sets, the following fact will be needed:

(1.13) Lemma. The condition supp $\mathscr{K} \underset{\mu}{=} X$ is satisfied if and only if

$$
1_{C} g \underset{\mathscr{H}}{\sim} 0 \text { for all } C \in \mathfrak{A} \text { implies } g_{\mu}^{=} 0 .
$$

Proof. 1. Assume first supp $\mathscr{K} \underset{\mu}{=} X$ and let $g \in \mathscr{L}_{\infty}(\mu)$ meet the requirement in $(*)$, i.e.

$$
\int_{C} f g d \mu=0 \text { for all } f \in \mathscr{K} \text { and } C \in \mathfrak{A} .
$$

Then $f g$ vanishes modulo $\mu$, hence

$$
\{g=0\} \supset_{\mu}\{f \neq 0\} \text { for all } f \in \mathscr{K},
$$

which implies

$$
\{g=0\} \underset{\mu}{\supset} \operatorname{supp} \mathscr{K} \underset{\mu}{=} X .
$$

2. Conversely, consider the function $g=1_{X \backslash \text { supp } \mathscr{K}}$, which obviously meets the requirement in $(*)$; therefore $g \underset{\mu}{=} 0$ or equivalently $\operatorname{supp} \mathscr{K} \underset{\mu}{=} X$.

Now denote by $\mathscr{G} / \mathscr{K}$ the quotient space of $\mathscr{G}$ with respect to $\tilde{\mathscr{H}}$, which is again convex. Then Satz 3.4 of [7], treating the marginal case, has the following analogue:

(1.14) Theorem. Let $\mathscr{K}$ satisfy $\operatorname{supp} \mathscr{K} \underset{\mu}{=} X$. Then a set $A \in \mathfrak{A}$ belongs to $\mathfrak{D}(\mathscr{K})$ if and only if $1_{A}$ is an extreme point of $\mathscr{G} / \mathscr{K}$.

Proof. 1. Assume first $A \in \mathfrak{D}(\mathscr{K})$ and consider an equivalence

$$
1_{A} \vartheta_{\mathscr{K}} \vartheta_{1}+\vartheta_{2} g_{2} \text { with } \vartheta_{i}>0, \vartheta_{1}+\vartheta_{2}=1 \text { and } g_{i} \in \mathscr{G} \text {. }
$$

Together this implies $g_{i} \underset{\mu}{=} 1_{A}$, hence $g_{i} \underset{\mathscr{K}}{\sim} 1_{A}$ as well.

2. Conversely, let $1_{A}$ be an extreme point of $\mathscr{G} / \mathscr{K}$ and consider an equivalence $1_{A} \underset{\mathscr{K}}{\widetilde{G}} g \in \mathscr{G}$. If $B \in \mathfrak{A}$ is any subset of $X \backslash A$, this yields

$$
1_{A} \underset{\mathscr{K}}{ } \frac{1}{2}\left(1_{A}+1_{B} g\right)+\frac{1}{2} 1_{X \backslash B} g .
$$

The right-hand side is a convex combination in $\mathscr{G} / \mathscr{K}$, hence

$$
1_{A} \tilde{\mathscr{R}} 1_{A}+1_{B} g \text { for all } B \in \mathfrak{A} \text { with } B \subset X \backslash A
$$

or, $B$ replaced by $C \cap(X \backslash A)$, equivalently

$$
1_{C}\left(1_{X \backslash A} g\right) \underset{\mathscr{H}}{\sim} 0 \text { for all } C \in \mathfrak{A} \text {. }
$$

Since the condition supp $\mathscr{K} \underset{\mu}{=} X$ is satisfied, (1.13) applies with the result $1_{X \backslash A} g{ }_{\mu}^{=} 0$. But it follows as in the proof of (1.5) that $1_{X \backslash A}$ is an extreme point 
of $\mathscr{G} / \mathscr{K}$ as well. Since $1_{X \backslash A} \tilde{\mathscr{K}} 1-g \in \mathscr{G}$, this implies similarly $1_{A}(1-g) \underset{\mu}{=} 0$. When combined, the last two equations yield $g_{\mu}^{=} 1_{A}$, proving $A \in \mathfrak{D}(\mathscr{K})$.

The case $\mu \neq 0$ and $\mathscr{K}=\{0\}$ shows at once that the condition supp $\mathscr{K}=\underset{\mu}{=} X$ is essential for the sufficiency in this criterion. Moreover, this condition being satisfied, there are no further functions $g \in \mathscr{G}$ yielding an extreme point of $\mathscr{G} / \mathscr{K}$. Indeed, consider the set

$$
A=\{\varepsilon<g<1-\varepsilon\} \quad \text { with } \varepsilon>0 \text { arbitrary. }
$$

For any $B \in \mathfrak{A}$ satisfying $B \subset A$ this yields

$$
g \underset{\mathscr{K}}{2} \frac{1}{2}\left(g-\varepsilon 1_{B}\right)+\frac{1}{2}\left(g+\varepsilon 1_{B}\right) \text { with } g \pm \varepsilon 1_{B} \in \mathscr{G},
$$

hence by the hypothesis

$$
g-\varepsilon 1_{B} \underset{\mathscr{K}}{\tilde{K}} g+\varepsilon 1_{B} \quad \text { for all } B \in \mathfrak{A} \text { with } B \subset A
$$

or equivalently

$$
1_{C} 1_{A} \underset{\mathscr{K}}{\sim} 0 \text { for all } C \in \mathfrak{A} .
$$

By (1.13) this implies $1_{A} \underset{\mu}{=}$, which for $\varepsilon \rightarrow 0$ proves $\mu(0<g<1)=0$.

\section{MAIN RESULtS}

The following characterization of $\mathscr{K}$-determined sets is of central importance and will allow an explicit criterion:

(2.1) Theorem. Let $h \in \mathscr{L}_{1}(\mu)$ be strictly positive. Then a set $A \in \mathfrak{A}$ belongs to $\mathfrak{D}(\mathscr{K})$ if and only if

$$
\inf _{f \in \mathscr{K}}\left(\int_{A}(h-f)^{+} d \mu+\int_{X \backslash A}(h+f)^{+} d \mu\right)=0 .
$$

Proof. 1. For fixed $A \in \mathfrak{A}$, due to the duality $\mathscr{L}_{1}^{*}(\mu)=\mathscr{L}_{\infty}(\mu)$, there is a bijection between the functions $g \in \mathscr{L}_{\infty}(\mu)$ such that

$$
\begin{aligned}
& g \underset{\mathscr{K}}{\widetilde{1}} 1_{A}, \\
& 0 \underset{\mu}{ } g \leq 1
\end{aligned}
$$

and the continuous linear functionals $I$ on $\mathscr{L}_{1}(\mu)$ such that

$$
\begin{gathered}
I(f)=\int_{A} f d \mu \text { for } f \in \mathscr{K}, \\
0 \leq I(f) \leq \int_{X} f d \mu \quad \text { for } 0 \leq f \in \mathscr{L}_{1}(\mu),
\end{gathered}
$$

where the continuity of $I$ is actually a consequence of condition $\left(2^{\prime}\right)$. By means of the functional

$$
J(f)=\int_{X} f^{+} d \mu \text { for } f \in \mathscr{L}_{1}(\mu)
$$


which is obviously positively homogeneous and subadditive, condition $\left(2^{\prime}\right)$ can be replaced by

$$
I(f) \leq J(f) \text { for all } f \in \mathscr{L}_{1}(\mu) .
$$

Indeed, this inequality implies $\left(2^{\prime}\right)$ due to

$$
I(f) \leq J(f)=0 \text { whenever } f \leq 0,
$$

while the converse is trivial.

2. Therefore, $A$ is $\mathscr{K}$-determined if and only if the linear functional

$$
I_{A}(f)=\int_{A} f d \mu \text { for } f \in \mathscr{L}_{1}(\mu)
$$

is the unique $J$-dominated extension of its restriction to $\mathscr{K}$. But this means that each such extension $I$ satisfies

$$
I\left(\left(1_{A}-1_{X \backslash A}\right) h\right)=I_{A}(h) .
$$

Indeed, due to the strict positivity of $h$, this equation implies $g=1_{A}$ for the function $g \in \mathscr{G}$ associated with $I$.

3. Now start the proof of the Hahn-Banach theorem by adjoining to $\mathscr{K}$ in the first step the function

$$
f_{0}=\left(1_{A}-1_{X \backslash A}\right) h \in \mathscr{L}_{1}(\mu) .
$$

Then it turns out that the admissible values for $I\left(f_{0}\right)$ reduce to $I_{A}(h)$ if and only if

$$
\sup _{f \in \mathscr{K}}\left(I_{A}(f)-J\left(f-f_{0}\right)\right)=I_{A}(h)=\inf _{f \in \mathscr{K}}\left(J\left(f+f_{0}\right)-I_{A}(f)\right) .
$$

By inserting $J$ and $I_{A}$ explicitly the first equation is easily transformed into the asserted condition, while the second one becomes

$$
\inf _{f \in \mathscr{K}}\left(\int_{A}(h+f)^{-} d \mu+\int_{X \backslash A}(h-f)^{-} d \mu\right)=0
$$

and is seen to be no condition at all by choosing $f=0$.

It is a natural question to ask whether the infimum in (2.1) is attained. In general this is not the case, because otherwise there would exist a function $f \in \mathscr{K}$ such that

$$
A \underset{\mu}{=}\{f \geq h\} \quad \text { and } \quad X \backslash A \underset{\mu}{=}\{f \leq-h\},
$$

a requirement even stronger than $A \in \mathfrak{S}(\mathscr{K})$.

An immediate consequence of $(2.1)$ is the following:

(2.2) Corollary. For each set $A \in \mathfrak{D}(\mathscr{K})$ there exists a subspace $\mathscr{K}_{0}$ of $\mathscr{K}$, generated by a countable basis, such that $A$ is already $\mathscr{K}_{0}$-determined.

Proof. Simply choose as basis a sequence of functions $f_{n} \in \mathscr{K}$ for which the crucial sum in (2.1) converges to its infimum 0 .

This result should be compared with the fact that a set $A \in \mathfrak{S}(\mathscr{K})$ is obviously $\mathscr{K}_{0}$-determined even for an appropriate subspace $\mathscr{K}_{0}$ of dimension 1 .

By means of the criterion (2.1) it is possible to decide by which "hull" $\mathscr{K}^{*}$ the subspace $\mathscr{K}$ has to be replaced in (1.6) in order to yield all $\mathscr{K}$-determined 
sets. Here, the crucial point consists in a generalization of the usual notion of weak convergence in $\mathscr{L}_{1}(\mu)$ to limits not contained in this space.

To include all monotone sequences, infinite values are allowed in the following extension:

(2.3) Definition. Let $f_{n}, n \in \mathbb{N}$, be a sequence in $\mathscr{L}_{1}(\mu)$ and $f^{*} \in \widetilde{\mathscr{L}}_{0}(\mu)$ be arbitrary. Then $f_{n} \rightsquigarrow f^{*}$, i.e. $f_{n}$ converges "weakly" to $f^{*}$, if

$$
\int_{X} f_{n} g d \mu \rightarrow \int_{X} f^{*} g d \mu
$$

for all $g \in \mathscr{L}_{\infty}(\mu)$ such that the right-hand side is well defined, i.e. at least one of the integrals $\int_{X}\left(f^{*} g\right)^{-} d \mu$ and $\int_{X}\left(f^{*} g\right)^{+} d \mu$ is finite.

For a first justification of this definition it will be shown that

$$
f_{1 n} \leq f_{2 n} \text { and } f_{i n} \rightsquigarrow f_{i}^{*} \text { implies } f_{1}^{*} \leq f_{\mu}^{*} .
$$

Indeed, the inequality is obvious on the set $\left\{f_{1}^{*} \leq 0 \leq f_{2}^{*}\right\}$ and follows on the sets $\left\{f_{1}^{*} \leq 0, f_{2}^{*} \leq 0\right\}$ and $\left\{f_{1}^{*} \geq 0, f_{2}^{*} \geq 0\right\}$ by choosing indicator functions for $g$ and passing to the limit, taking into account that $\mu$ is $\sigma$-finite. Finally, $A=\left\{f_{1}^{*}>0>f_{2}^{*}\right\}$ has measure zero, due to

$$
0 \leq \int_{A} f_{1}^{*} d \mu=\lim _{n \rightarrow \infty} \int_{A} f_{1 n} d \mu \leq \lim _{n \rightarrow \infty} \int_{A} f_{2 n} d \mu=\int_{A} f_{2}^{*} d \mu \leq 0 .
$$

This proves the assertion, which in particular implies that in case of convergence the limit is unique. Moreover, this extended weak convergence is obviously compatible with scalar multiplication.

The scope of $(2.3)$ is seen by the easily established fact that each function $f^{*} \in \widetilde{\mathscr{L}}_{0}(\mu)$ is a weak limit of functions $f_{n} \in \mathscr{L}_{1}(\mu)$ or by the example $n f \leadsto$ $f^{*}$ with $f^{*}(x)=-\infty, 0,+\infty$ as $f(x)<0,=0,>0$ for each function $f \in \mathscr{L}_{1}(\mu)$.

It is clear that the convergence condition in (2.3) can be reduced to

$$
\int_{X} f_{n} g d \mu \rightarrow \int_{X} f^{*} g d \mu
$$

for all $g \in \mathscr{G}$ such that the support of $g$ is contained in $\left\{f^{*} \leq 0\right\}$ or $\left\{f^{*} \geq 0\right\}$. It is an open problem, however, even in the case of a countable space $X$, whether $g$ can be restricted further to indicator functions, as is true for the usual notion of weak convergence.

The hull of $\mathscr{K}$ with respect to this extended weak convergence will be denoted by a special symbol:

(2.4) Definition. $\mathscr{K}^{*}$ is the class of all functions $f^{*} \in \widetilde{\mathscr{L}}_{0}(\mu)$ such that $f_{n}$ m $f^{*}$ for some sequence $f_{n} \in \mathscr{K}, n \in \mathbb{N}$.

Clearly, $\mathscr{K}^{*}$ is again stable under scalar multiplication. Moreover, passing from $\mathscr{K}$ to $\overline{\mathscr{K}}$ makes no difference, because for functions $f_{n}, f_{n}^{\prime} \in \mathscr{L}_{1}(\mu)$ certainly

$$
f_{n} \rightsquigarrow f^{*} \text { and }\left\|f_{n}-f_{n}^{\prime}\right\| \rightarrow 0 \quad \text { implies } f_{n}^{\prime} \rightsquigarrow f^{*} .
$$

After these preliminaries the main characterization of the class $\mathfrak{D}(\mathscr{K})$ can be established: 
(2.5) Theorem. A set $A \in \mathfrak{A}$ belongs to $\mathfrak{D}(\mathscr{K})$ if and only if

$$
A \underset{\mu}{=}\left\{f^{*}>0\right\} \text { and } X \backslash A \underset{\mu}{=}\left\{f^{*}<0\right\}
$$

for some function $f^{*} \in \mathscr{K}^{*}$.

Proof. 1. Assume first $A \in \mathfrak{D}(\mathscr{K})$ and apply (2.1) to obtain a sequence $f_{n} \in$ $\mathscr{K}, n \in \mathbb{N}$, such that

$$
\sum_{n \in \mathbb{N}}\left(\int_{A}\left(h-f_{n}\right)^{+} d \mu+\int_{X \backslash A}\left(h+f_{n}\right)^{+} d \mu\right)<\infty
$$

for a fixed strictly positive function $h \in \mathscr{L}_{1}(\mu)$. Interchanging summation and integration yields

$$
\sum_{n \in \mathbb{N}}\left(h-f_{n}\right)^{+}<\infty \text { on } A \text { and } \sum_{n \in \mathbb{N}}\left(h+f_{n}\right)^{+}<\infty \text { on } X \backslash A,
$$

and this implies easily

$$
\sum_{n \in \mathbb{N}} f_{n}=+\infty \text { on } A \text { and } \sum_{n \in \mathbb{N}} f_{n}=-\infty \text { on } X \backslash A,
$$

always understood modulo $\mu$. Moreover, the estimates $f_{n}^{-} \leq\left(h-f_{n}\right)^{+}$and $f_{n}^{+} \leq\left(h+f_{n}\right)^{+}$lead to

$$
\int_{A}\left(\sum_{n \in \mathbb{N}} f_{n}^{-}\right) d \mu<\infty \text { and } \int_{X \backslash A}\left(\sum_{n \in \mathbb{N}} f_{n}^{+}\right) d \mu<\infty .
$$

When combined, (1) and (2) yield without difficulty

$$
\mathscr{K} \ni f_{1}+\cdots+f_{n} \rightsquigarrow f^{*}=\sum_{n \in \mathbb{N}} f_{n},
$$

hence $f^{*}$ is contained in $\mathscr{K}^{*}$ and has the required property.

2. To prove the converse, let $A$ be represented by means of $f^{*}$ with $\mathscr{K} \ni$ $f_{n} \leadsto f^{*}$ and assume $1_{A} \underset{\mathscr{K}}{\tilde{H}} g \in \mathscr{G}$. Then

$$
\int_{X} f_{n} 1_{A} d \mu=\int_{X} f_{n} g d \mu
$$

and this is equivalent to

$$
\int_{X} f_{n}\left(1_{A}-g\right)^{+} d \mu=\int_{X} f_{n}\left(g-1_{A}\right)^{+} d \mu,
$$

where the supports of $\left(1_{A}-g\right)^{+}$and $\left(g-1_{A}\right)^{+}$are contained in $\left\{f^{*}>0\right\}$ and $\left\{f^{*}<0\right\}$, respectively. Therefore the passage $n \rightarrow \infty$ is possible and results in

$$
\int_{X} f^{*}\left(1_{A}-g\right)^{+} d \mu=\int_{X} f^{*}\left(g-1_{A}\right)^{+} d \mu .
$$

But the two integrals are of opposite sign, hence

which means

$$
\int_{X} f^{*}\left(1_{A}-g\right)^{+} d \mu=0=\int_{X} f^{*}\left(g-1_{A}\right)^{+} d \mu,
$$

$$
g \underset{\mu}{\geq} 1_{A} \text { on }\left\{f^{*}>0\right\} \text { and } g \leq_{\mu} 1_{A} \text { on }\left\{f^{*}<0\right\},
$$

combining to $g_{\mu}^{=} 1_{A}$, as had to be shown. 
It is established in part 1 of the proof that the function $f^{*} \in \mathscr{K}^{*}$ representing $A$ actually can be restricted to the values $\pm \infty$. As will be seen in (3.3), it does not suffice to consider only finite-valued functions $f^{*}$. This example, moreover, will show that the hull $\mathscr{K}^{*}$ cannot simply be replaced by the set of all limits of monotone sequences in $\mathscr{K}$. Finally, it should be mentioned that $A \in \mathfrak{D}(\mathscr{K})$ is already a consequence of representations

$$
A \underset{\mu}{=}\left\{f_{1}^{*}>0\right\} \quad \text { and } X \backslash A \underset{\mu}{=}\left\{f_{2}^{*}<0\right\}
$$

with arbitrary functions $f_{i}^{*} \in \mathscr{K}$. Indeed, in this case $f^{*}=f_{1}^{*}+f_{2}^{*}$ is well defined and $\mathscr{K} \ni f_{i n} \leadsto f_{i}^{*}$ implies $\mathscr{K} \ni f_{1 n}+f_{2 n} \rightsquigarrow f^{*}$, hence $f^{*}$ is contained in $\mathscr{K}^{*}$ and in addition has the required property.

To conclude this section with a simple application, consider the question under what condition $X$ itself is $\mathscr{K}$-determined. According to (2.5) and the subsequent remark this holds if and only if there exists a sequence $f_{n} \in \mathscr{K}$, $n \in \mathbb{N}$, with the property

$$
\lim _{n \rightarrow \infty} \int_{A} f_{n} d \mu=\infty \quad \text { whenever } \mu(A)>0 .
$$

\section{Generalized SEPARATED SETS}

The passage from $\mathscr{K}$ in (1.6) to $\mathscr{K}^{*}$ in (2.5) is somewhat involved. It is, therefore, important to find classes lying between $\mathfrak{S}(\mathscr{K})$ and $\mathfrak{D}(\mathscr{K})$. As outlined in the introduction this suggests a natural generalization of the concept of $\mathscr{K}$-separated sets.

To this end lexicographic order will be needed:

(3.1) Definition. For any ordinal $\gamma>0$ the linear space $\mathbb{R}^{\gamma}=\prod_{\alpha<\gamma} \mathbb{R}$ is totally ordered by the relation

$$
\left\{\begin{array}{l}
\left(u_{\alpha}, \alpha<\gamma\right) \preceq\left(v_{\alpha}, \alpha<\gamma\right) \text { if and only if } \\
B=\left\{\alpha: u_{\alpha} \neq v_{\alpha}\right\}=\varnothing \text { or } u_{\beta}<v_{\beta} \text { for } \beta=\min B .
\end{array}\right.
$$

This order is compatible with the linear structure, in particular

$$
u \succeq v \text { if and only if } u-v \succeq 0,
$$

where the symbols $\succeq$ resp. $\prec$ and $\succ$ have the usual meaning.

To ensure measurability, actually a restriction to countable ordinals is necessary in the sequel. Beginning with $\mathfrak{S}(\mathscr{K})$, this yields the following hierarchy:

(3.2) Definition. If $\gamma>0$ is a countable ordinal, the class $\mathfrak{S}_{\gamma}(\mathscr{K})$ consists of all sets $A \in \mathfrak{A}$ that are " $\mathscr{K}$-separated of order $\gamma$ ", i.e.

$$
A \underset{\mu}{=}\left\{\left(f_{\alpha}, \alpha<\gamma\right) \succ 0\right\} \quad \text { and } X \backslash A \underset{\mu}{=}\left\{\left(f_{\alpha}, \alpha<\gamma\right) \prec 0\right\}
$$

for suitable functions $f_{\alpha} \in \mathscr{K}, \alpha<\gamma$.

As (1.6) this notion is symmetric in $A$ and its complement. Extending $f_{\alpha}, \alpha<\gamma$, by copies of $f=0$ shows that the class $\mathfrak{S}_{\gamma}(\mathscr{K})$ increases when $\gamma$ increases. Actually, the map $\gamma \mapsto \mathfrak{S}_{\gamma}(\mathscr{K})$ may strictly increase at each ordinal. Thus, in particular, $\gamma \leq \omega_{0}$ (the first infinite ordinal) is really a restriction, i.e. (3.2) cannot be confined to ordinary sequences $f_{n} \in \mathscr{K}, n \in \mathbb{N}$. To exemplify this, a more elaborate construction is required: 
(3.3) Example. Let $\gamma>0$ be a countable ordinal and $X \subset \mathbb{R}^{\gamma}$ consist of all $x=\left(x_{\alpha}, \alpha<\gamma\right)$ such that $x_{\alpha} \in \mathbb{Z}$ for every $\alpha$ and in addition $x_{\alpha} \neq 0$ for at least one and at most finitely many indices. Moreover, let $\mu$ be a strictly positive measure on the countable space $X$ and choose for $\mathscr{K}$ the closed linear space generated by the projections $\pi_{\alpha}: x \mapsto x_{\alpha}, \alpha<\gamma$, which are contained in $\mathscr{L}_{1}(\mu)$ as soon as $\mu$ integrates the finite-valued function $f=\sum_{\alpha<\gamma}\left|\pi_{\alpha}\right|$. Then it turns out that

$$
A=\left\{\left(\pi_{\alpha}, \alpha<\gamma\right) \succ 0\right\} \in \mathfrak{S}_{\gamma}(\mathscr{K}) \backslash \bigcup_{\beta<\gamma} \mathfrak{S}_{\beta}(\mathscr{K}) .
$$

Indeed, $A \in \mathfrak{S}_{\gamma}(\mathscr{K})$ follows from $0 \notin X$, and it remains to show that a representation

$$
A=\left\{\left(f_{\alpha}, \alpha<\beta\right) \succ 0\right\} \quad \text { and } \quad X \backslash A=\left\{\left(f_{\alpha}, \alpha<\beta\right) \prec 0\right\}
$$

with $f_{\alpha} \in \mathscr{K}$ and $\beta<\gamma$ leads to a contradiction. To this end observe first that norm convergence in $\mathscr{L}_{1}(\mu)$ implies pointwise convergence on $X$ and thus each function $f \in \mathscr{K}$ is of the form

$$
f=\sum_{\alpha<\gamma} t_{\alpha} \pi_{\alpha} \text { with } t_{\alpha} \in \mathbb{R}
$$

where the sum in fact is finite, if $f$ is evaluated at any $x \in X$. Now from

$$
A \in \sigma\left(f_{\alpha}, \alpha<\beta\right) \text { and } A \notin \sigma\left(\pi_{\alpha}, \alpha<\beta\right)
$$

it follows that there is a first $\alpha_{0}<\gamma$ such that $f_{\alpha_{0}}$ is not measurable with respect to $\sigma\left(\pi_{\alpha}, \alpha \leq \alpha_{0}\right)$. This implies in turn the existence of a first $\beta_{0}>\alpha_{0}$ such that

$$
f_{\alpha_{0}}=\sum_{\alpha \leq \alpha_{0}} t_{\alpha} \pi_{\alpha}+t_{\beta_{0}} \pi_{\beta_{0}}+\sum_{\beta_{0}<\alpha<\gamma} t_{\alpha} \pi_{\alpha}
$$

with $t_{\beta_{0}} \neq 0$. Consider, finally, for arbitrary $n \in \mathbb{Z}$ the point $x \in X$ with $x_{\alpha_{0}}=-1, x_{\beta_{0}}=n$, and $x_{\alpha}=0$ otherwise. Then $x \prec 0$, hence $x \notin A$ and thus $\left(f_{\alpha}(x), \alpha<\beta\right) \prec 0$. But this implies

$$
t_{\alpha_{0}} \cdot(-1)+t_{\beta_{0}} \cdot n \leq 0 \text { for all } n \in \mathbb{Z}
$$

in contradiction to $t_{\beta_{0}} \neq 0$.

Next, the statement of (1.7) can be extended:

(3.4) Theorem. $\mathfrak{S}_{\gamma}(\mathscr{K}) \subset \mathfrak{D}(\mathscr{K})$ for each countable ordinal $\gamma>0$.

Proof. 1. Assume without loss of generality $\mathscr{K}$ to be closed and let $A \in \mathfrak{S}_{\gamma}(\mathscr{K})$ be represented by $f_{\alpha} \in \mathscr{K}, \alpha<\gamma$, where $a=\sum_{\alpha<\gamma}\left\|f_{\alpha}\right\|<\infty$ may be achieved by taking suitable multiples. Choose real numbers $r_{\alpha}>0$ with $\sum_{\alpha<\gamma} r_{\alpha}<\infty$ and consider the functions

$$
f_{n}^{\prime}=\sum_{\alpha<\gamma} n^{s_{\alpha}} f_{\alpha} \text { with } s_{\alpha}=\sum_{\beta \geq \alpha} r_{\beta},
$$

which, due to the normalization of $f_{\alpha}, \alpha<\gamma$, belong to $\mathscr{K}$. The assertion will be established by proving $f_{n}^{\prime} \leadsto f^{*}$, where

$$
f^{*}=+\infty \text { on } A \text { and } f^{*}=-\infty \text { on } X \backslash A,
$$

and applying (2.5). 
2. Let for instance $g \in \mathscr{G}$ satisfy

$$
\{g>0\} \subset\left\{f^{*} \geq 0\right\} \text { and } \mu(g>0)>0 .
$$

Then it must be shown that

$$
\int_{X} f_{n}^{\prime} g d \mu=\int_{A} f_{n}^{\prime} g d \mu \rightarrow+\infty \text { for } n \rightarrow \infty .
$$

To this end partition $A=\left\{\left(f_{\alpha}, \alpha<\gamma\right) \succ 0\right\}$ into the sets

$$
A_{\beta}=\left\{f_{\alpha}=0 \text { for } \alpha<\beta, f_{\beta}>0\right\} \in \mathfrak{A}, \quad \beta<\gamma,
$$

and define

$$
\beta_{0}=\min \left\{\beta: \mu\left(A_{\beta} \cap\{g>0\}\right)>0\right\} .
$$

With the abbreviations $r=r_{\beta_{0}}$ and $s=s_{\beta_{0}}$ this yields the inequality

$$
\begin{aligned}
\int_{X} f_{n}^{\prime} g d \mu & =\sum_{\beta \geq \beta_{0}} \int_{A_{\beta}} f_{n}^{\prime} g d \mu \\
& =\sum_{\beta \geq \beta_{0}} \sum_{\alpha \geq \beta} n^{s_{\alpha}} \int_{A_{\beta}} f_{\alpha} g d \mu \\
& \geq n^{s} \int_{A_{\beta_{0}}} f_{\beta_{0}} g d \mu-\sum_{\alpha>\beta_{0}} n^{s_{\alpha}} \int_{X}\left|f_{\alpha}\right| d \mu \\
& \geq n^{s}\left(b-\frac{1}{n^{r}} a\right),
\end{aligned}
$$

where

$$
b=\int_{A_{\beta_{0}}} f_{\beta_{0}} g d \mu>0 .
$$

Thus the lower bound tends to $+\infty$, and the proof is completed.

At this point two questions left open in the context of (2.5) can be settled. Taking $\gamma=2$ in (3.3) yields

$$
\mathscr{K}=\left\{t_{0} \pi_{0}+t_{1} \pi_{1}: t_{i} \in \mathbb{R}\right\},
$$

and this set is closed with respect to pointwise limits in $\mathbb{R}$ as well as with respect to monotone limits in $\widetilde{\mathbb{R}}$. Since

$$
\mathfrak{S}_{1}(\mathscr{K}) \varsubsetneqq \mathfrak{S}_{2}(\mathscr{K}) \subset \mathfrak{D}(\mathscr{K}),
$$

this shows that restricting the definition of $\mathscr{K}^{*}$ to finite-valued limits or limits of monotone sequences in general is not possible.

Most interesting in the context of (3.4) is the question whether $\mathfrak{D}(\mathscr{K})$ is simply the union of the classes $\mathfrak{S}_{\gamma}(\mathscr{K}), \gamma<\omega_{1}$ (the first uncountable ordinal). This fails to hold even for countable spaces $X$ :

(3.5) Example. Endow $X=\mathbb{Z}$ with the measure $\mu$ defined by $\mu(\{n\})=2^{n}$ and consider the linear subspace

$$
\mathscr{K}=\left\{f \in \mathscr{L}_{1}(\mu): f(-n)=f(n)-f(n-1) \text { for } n \in \mathbb{N}\right\},
$$

which is clearly closed. Then

$$
\varnothing \in \mathfrak{D}(\mathscr{K}) \backslash \bigcup_{\gamma<\omega_{1}} \mathfrak{S}_{\gamma}(\mathscr{K}) .
$$


Indeed, $0 \underset{\mathscr{H}}{\sim} g \in \mathscr{G}$ implies $\int_{X} f_{n} g d \mu=0$ for

$$
f_{n}=1_{\{-n,+n\}}-1_{\{-n-1\}} \in \mathscr{K},
$$

which means explicitly

$$
2^{-n} g(-n)+2^{n} g(n)-2^{-n-1} g(-n-1)=0 \text { for } n \in \mathbb{N} .
$$

But, due to $g \geq 0$, this leads to $g(-n-1) \geq 2 g(-n)$ and, due to $g \leq 1$, to $g(-n)=0$ for $n \in \mathbb{N}$. Considering any function $f \in \mathscr{K}$ that is strictly positive for $n \geq 0$, it turns out that $g$ must in fact vanish everywhere, and thus $\varnothing$ is indeed $\mathscr{K}$-determined. The assumption that $\varnothing$ (or $X$ ) is $\mathscr{K}$-separated of some order $\gamma$, however, implies the existence of functions $f_{\alpha}^{0} \in \mathscr{K}, \alpha<\gamma$, such that

$$
\left(f_{\alpha}^{0}(x), \alpha<\gamma\right) \succ 0 \text { for all } x \in X .
$$

But the only nonnegative function in $\mathscr{K}$ is $f=0$, because

$$
f(n)=f(n-1)+f(-n) \geq f(n-1) \text { for } n \in \mathbb{N},
$$

in view of the integrability, implies $f(n)=0$ for $n \geq 0$, hence also $f(n)=$ 0 for $n<0$. By recursion this yields $f_{\alpha}^{0}=0$ for all $\alpha<\gamma$ and thus a contradiction.

Incidentally, it should be mentioned that this example can be shown to contradict Theorem VI.2.2 in [11], stating that through every extreme point of the closed convex hull of the range of a vector measure passes a supporting hyperplane.

As already mentioned in the introduction the approach in (1.6) and (3.2) is closely related to asymmetric notions in the existing literature, using the term "additive" for historical reasons. Essentially, Kemperman in [8] studies the finite case and in [9] mentions the infinite case of the following notion:

(3.6) Definition. If $\gamma>0$ is a countable ordinal, a set $A \in \mathfrak{A}$ is called " $\mathscr{K}$ supported of order $\gamma "$, if

$$
A \underset{\mu}{=}\left\{\left(f_{\alpha}, \alpha<\gamma\right) \succ 0\right\} \quad \text { with } f_{\alpha} \in \mathscr{K}, \alpha<\gamma .
$$

The relation to (3.2) is easily established:

(3.7) Proposition. Let $\gamma>0$ be a countable ordinal. Then

(a) a set $A$ is $\mathscr{K}$-separated of order $\gamma$ if and only if $A$ and $X \backslash A$ are both $\mathscr{K}$-supported of order $\gamma$;

(b) if a set $A$ is $\mathscr{K}$-supported of order $\gamma$ and $\mathscr{K}$ contains a strictly positive function $f_{0}$, then $A$ is $\mathscr{K}$-separated of order $\gamma+1$.

Proof. (a) Since the condition clearly is necessary, assume

$$
A_{\mu}=\left\{\left(f_{\alpha}^{\prime}, \alpha<\gamma\right) \succ 0\right\} \quad \text { and } \quad X \backslash A \underset{\mu}{=}\left\{\left(f_{\alpha}^{\prime \prime}, \alpha<\gamma\right) \succ 0\right\}
$$

for suitable $f_{\alpha}^{\prime}, f_{\alpha}^{\prime \prime} \in \mathscr{K}$. Then it is immediate that

$$
f_{\alpha}=f_{\alpha}^{\prime}-f_{\alpha}^{\prime \prime} \in \mathscr{K} \quad \text { for } \alpha<\gamma
$$

yields a representation in the sense of (3.2). 
(b) If $A \underset{\mu}{=}\left\{\left(f_{\alpha}, \alpha<\gamma\right) \succ 0\right\}$ with $f_{\alpha} \in \mathscr{K}$ and $f_{\gamma}=-f_{0}$, then $f_{\alpha}$, $\alpha<\gamma+1$, yields again a representation in the sense of (3.2).

The case $\mathscr{K}=\{0\}$ shows at once that the additional assumption in part (b) of this proposition cannot be dispensed with.

The final result of this section concerns the special case of a finite-dimensional space $\mathscr{K}$. An application of (1.14) allows a simple proof of the following result, which corresponds to Theorem 4.16 in [8]:

Proposition. If $0<k=\operatorname{dim} \mathscr{K}<\infty$, then

$$
\mathfrak{D}(\mathscr{K})=\mathfrak{S}_{k}(\mathscr{K}) .
$$

Proof. 1. If $f_{i}, 0 \leq i<k$, is a basis of $\mathscr{K}$, the range

$$
R=\left\{\left(\int_{X} f_{i} g d \mu, 0 \leq i<k\right): g \in \mathscr{G}\right\}
$$

is a compact convex set in $\mathbb{R}^{k}$ by Alaoglu's theorem, and

$$
\varphi: \mathscr{G} / \mathscr{K} \ni g \mapsto\left(\int_{X} f_{i} g d \mu, 0 \leq i<k\right) \in R
$$

is a bijection that respects convex combinations. But, due to (1.11) and (3.4), supp $\mathscr{K} \underset{\mu}{=} X$ may be assumed, and thus (1.14) is available. It suffices, therefore, to characterize the extreme points of $R$.

2. Each of these points is obtained by first applying an orthogonal transformation $L$ to $\mathbb{R}^{k}$ and then taking the lexicographic maximum of $L[R]$ (see e.g. [6, p. 44]). But $L$ can be cancelled out, replacing the elements of the basis by $L\left(f_{i}\right)$, and it remains to find the set $A \in \mathfrak{A}$ maximizing $\left(\int_{A} f_{i} d \mu, 0 \leq i<k\right)$ in lexicographic order. Since this order relation is compatible with the linear structure, hence with integration, $A$ and $X \backslash A$ have to satisfy

$$
A \supset \supset_{\mu}\left\{\left(f_{i}, 0 \leq i<k\right) \succ 0\right\} \text { and } X \backslash A \supset \supset_{\mu}\left\{\left(f_{i}, 0 \leq i<k\right) \prec 0\right\} .
$$

Since $\left\{\left(f_{i}, 0 \leq i<k\right)=0\right\}$ is a null set, due to $\operatorname{supp} \mathscr{K} \underset{\mu}{=} X$, this means $A \in \mathfrak{S}_{k}(\mathscr{K})$, and the proof is completed.

As is clear from (3.3), the equation $\mathfrak{D}(\mathscr{K})=\mathfrak{S}_{\gamma}(\mathscr{K})$ in general need not hold for some $\gamma<k$. An exception, however, is the case

$$
\{f=0\} \underset{\mu}{=} \varnothing \text { or } X \text { for all } f \in \mathscr{K},
$$

where obviously $\mathfrak{S}_{k}(\mathscr{K})=\mathfrak{S}(\mathscr{K})$.

\section{Almost Determined SETS}

As shown in the preceding section the class $\mathfrak{D}(\mathscr{K})$ is not necessarily exhausted by the classes $\mathfrak{S}_{\gamma}(\mathscr{K})$. This raises the question whether $\mathscr{K}$-determined sets can at least be approximated by $\mathscr{K}$-separated sets. To treat this problem, the $\sigma$-algebra $\mathfrak{A}$ or, more generally, the space $\mathscr{L}_{0}(\mu)$ has to be topologized first. The adequate topology is induced by convergence in measure, denoted by $\overrightarrow{0}$ in the sequel, which is defined via convergence modulo $\mu$, denoted simply by $\rightarrow$, 
as usual: a sequence in $\mathscr{L}_{0}(\mu)$ converges in measure, if each subsequence contains a subsubsequence converging modulo $\mu$. Since this measure is $\sigma$-finite, the topology is also induced by the metric

$$
d\left(e_{1}, e_{2}\right)=\int_{X}\left|e_{1}-e_{2}\right| \wedge h d \mu \text { for } e_{i} \in \mathscr{L}_{0}(\mu),
$$

where $h$ is any strictly positive function in $\mathscr{L}_{1}(\mu)$. In a natural way $\mathfrak{A}$ is topologized as a-closed-subspace of $\mathscr{L}_{0}(\mu)$.

Before investigating the approximation problem stated above, the topological structure of $\mathfrak{D}(\mathscr{K})$ has to be clarified. The first result is very satisfactory:

(4.1) Proposition. If $\operatorname{dim} \mathscr{K}<\infty$, then $\mathfrak{D}(\mathscr{K})$ is closed in $\mathfrak{A}$.

Proof. 1. Multiply the basic measure space $(X, \mathfrak{A}, \mu)$ by a nonatomic measure space $\left(X^{\prime}, \mathfrak{A}^{\prime}, \mu^{\prime}\right)$ with $0<\mu^{\prime}\left(X^{\prime}\right)<\infty$, to obtain a $\sigma$-finite product space $(\widetilde{X}, \tilde{\mathfrak{A}}, \tilde{\mu})$ that is again nonatomic. Next, denote by $\pi$ the projection from $\widetilde{X}$ to $X$ and transform $\mathscr{K} \subset \mathscr{L}_{1}(\mu)$ into $\widetilde{\mathscr{K}}=\{f \circ \pi: f \in \mathscr{K}\} \subset \mathscr{L}_{1}(\tilde{\mu})$, which is again a finite-dimensional space. Then it is an immediate consequence of (1.4) that

$$
\tilde{A} \in \mathfrak{D}(\widetilde{\mathscr{K}}) \quad \text { if and only if } \quad \tilde{A}=\underset{\tilde{\mu}}{=} A \times X^{\prime} \text { with } A \in \mathfrak{D}(\mathscr{K}) .
$$

Combined with

$$
A_{n} \underset{0}{\rightarrow} A \text { in } \mathfrak{A} \text { if and only if } A_{n} \times X^{\prime} \underset{0}{\vec{a}} A \times X^{\prime} \text { in } \tilde{\mathfrak{A}},
$$

this shows that it suffices to consider the nonatomic case.

2. Assume now without loss of generality $k=\operatorname{dim} \mathscr{K}>0$ and use the same notation as in the proof of (3.8). Then the mapping

$$
\psi: \mathscr{G} \ni g \mapsto\left(\int_{X} f_{i} g d \mu, 0 \leq i<k\right) \in R
$$

is easily seen to be continuous and according to [19] (where a gap in [1] is closed) to be open as well. The proof can therefore be completed by referring to the following simple topological fact: if $\psi: U \rightarrow V$ is continuous and open, the set

$$
U_{0}=\left\{u \in U: \psi\left(u^{\prime}\right)=\psi(u) \text { implies } u^{\prime}=u\right\}
$$

is closed in $U$, whenever this is a Hausdorff space. Thus the corresponding subset $\mathscr{G}_{0}$ is closed in $\mathscr{G}$. Restricted to indicator functions, this proves $\mathfrak{D}(\mathscr{K})$ to be closed in $\mathfrak{A}$.

As is seen already from (1.12), the condition $\operatorname{dim} \mathscr{K}<\infty$ is essential for this result. More interesting, however, is the following situation:

(4.2) Example. Let $X$ be the product of the spaces $X_{i}=\mathbb{Z}, 1 \leq i \leq 3$, and denote by $\pi_{i}$ the associated projections. In $X$ consider the points

$$
x^{1 k}=\left(a_{k},-a_{k-1},-a_{k-1}\right), \ldots, x^{3 k}=\left(-a_{k-1},-a_{k-1}, a_{k}\right),
$$

where $a_{k}=2^{k}-1$ for $k \geq 0$, and define a normed measure $\nu$ by

$$
\nu\left(\left\{x^{i k}\right\}\right)=\frac{1}{3} 2^{-k} \text { for } 1 \leq i \leq 3 \text { and } k \in \mathbb{N} .
$$

Then the marginals $\mu_{i}=\pi_{i}(\mu)$ are given by

$$
\nu_{i}\left(\left\{ \pm a_{k}\right\}\right)=\frac{1}{3} 2^{-k} \text { for } 1 \leq i \leq 3 \text { and } k \geq 0,
$$


i.e. the measures $\nu_{i}$ are symmetric with respect to the origin, although $\nu$ itself is supported by the hyperplane $\sum_{1 \leq i \leq 3} \pi_{i}=1$ (this is a slight simplification of a counterexample in [20, p. 155]; related examples can be found in [2] and [18]). Now let $\mu$ be the symmetrization of $\nu$, having marginals $\mu_{i}=\nu_{i}$ and being supported by the hyperplanes $\sum_{1 \leq i \leq 3} \pi_{i}= \pm 1$, and consider

$$
\mathscr{K}=\left\{\sum_{1 \leq i \leq 3} f_{i} \circ \pi_{i}: f_{i} \in \mathscr{L}_{1}\left(\mu_{i}\right)\right\} .
$$

Then $\mathfrak{D}(\mathscr{K})$ fails to be closed, because

$$
\begin{aligned}
A_{n} & =\left\{\sum_{1 \leq i \leq 3}\left(\pi_{i} \wedge n\right)>0\right\} \in \mathfrak{D}(\mathscr{K}) \text { for } n \in \mathbb{N}, \\
A & =\left\{\sum_{1 \leq i \leq 3} \pi_{i}>0\right\} \notin \mathfrak{D}(\mathscr{K}),
\end{aligned}
$$

although $A_{n} \uparrow A$. Indeed, the first assertion follows from (2.5), because

$$
f_{n}^{*}=\sum_{1 \leq i \leq 3}\left(\pi_{i} \wedge n\right)-\frac{1}{2}
$$

as the limit of a decreasing sequence in $\mathscr{K}$ satisfies $f_{n}^{*} \in \mathscr{K}^{*}$ and

$$
A_{n}=\left\{f_{n}^{*}>0\right\} \text { and } X \backslash A_{n}=\left\{f_{n}^{*}<0\right\},
$$

while the second assertion is a consequence of $A \tilde{\mathscr{K}} X \backslash A$.

Moreover, this example answers in the negative the question whether a representation as in (2.5) is sufficient for $A \in \mathfrak{D}(\mathscr{K})$, if $f^{*}$ is chosen from the monotone class generated by $\mathscr{K}$ rather than from $\mathscr{K}^{*}$.

Incidentally, it is of some interest that the subspace $\mathscr{K}$ fails to be closed (compare a related counterexample in [14]). Indeed, choose real numbers $b_{k}$ with $b_{k} \downarrow 0$ and $\sum_{k \geq 0} b_{k}=\infty$ and define functions $e_{n}$ and $e$ on $\mathbb{Z}$ by

$$
\begin{aligned}
e_{n}\left( \pm a_{k}\right) & =(-2)^{k} b_{k} \quad \text { for } 0 \leq k<n \text { (0 otherwise) } \\
e\left( \pm a_{k}\right) & =(-2)^{k} b_{k} \quad \text { for } k \geq 0 \text { (0 otherwise), }
\end{aligned}
$$

satisfying $e_{n} \in \mathscr{L}_{1}\left(\mu_{1}\right)$ and $e \notin \mathscr{L}_{1}\left(\mu_{i}\right)$. Then

$$
f_{n}=\sum_{1 \leq i \leq 3} e_{n} \circ \pi_{i} \in \mathscr{K},
$$

and a simple computation yields

$$
\left\|f_{n}-f\right\|=2 b_{n} \rightarrow 0 \text { with } f=\sum_{1 \leq i \leq 3} e \circ \pi_{i} .
$$

Now assume $f \in \mathscr{K}$ and symmetrize with respect to $i$ to obtain

$$
f=\sum_{1 \leq i \leq 3} e^{\prime} \circ \pi_{i} \quad \text { with } e^{\prime} \in \mathscr{L}_{1}\left(\mu_{i}\right) \text {. }
$$


But $e^{\prime}$ can be symmetrized with respect to the origin as well, and then $e^{\prime \prime}=e^{\prime}-e$ is a solution of the homogeneous equation

$$
\sum_{1 \leq i \leq 3} e^{\prime \prime} \circ \pi_{i}=0 \quad \text { with } e^{\prime \prime}\left(-x_{i}\right)=e^{\prime \prime}\left(+x_{i}\right),
$$

which is easily solved, resulting in

$$
e^{\prime \prime}\left( \pm a_{k}\right)=(-2)^{k} e^{\prime \prime}(0) \text { for } k \geq 0
$$

This yields

$$
e^{\prime}\left( \pm a_{k}\right)=(-2)^{k}\left(b_{k}+e^{\prime \prime}(0)\right) \text { for } k \geq 0,
$$

contradicting the assumption $e^{\prime} \in \mathscr{L}_{1}\left(\mu_{i}\right)$ for every choice of $e^{\prime \prime}(0)$.

After this digression the topological structure of $\mathfrak{D}(\mathscr{K})$ has to be studied without dimension restrictions. In view of (1.14) the following general result is not surprising:

(4.3) Proposition. $\mathfrak{D}(\mathscr{K})$ is of type $G_{\delta}$ in $\mathfrak{A}$.

Proof. Given $h$ as in (2.1), the function

$$
\varphi_{f}(A)=\int_{A}(h-f)^{+} d \mu+\int_{X \backslash A}(h+f)^{+} d \mu
$$

is continuous on $\mathfrak{A}$ for every $f \in \mathscr{L}_{1}(\mu)$, hence $\varphi(A)=\inf _{f \in \mathscr{K}} \varphi_{f}(A)$ is upper semicontinuous on $\mathfrak{A}$. Thus

$$
\{A \in \mathfrak{A}: \varphi(A)=0\}=\bigcap_{n \in \mathbb{N}}\left\{A: \varphi(A)<\frac{1}{n}\right\}
$$

is of type $G_{\delta}$, and the assertion follows from (2.1).

Since $\mathfrak{D}(\mathscr{K})$ need not be closed, the following notion is natural:

(4.4) Definition. A set $A \in \mathfrak{A}$ is called "almost $\mathscr{K}$-determined", if it belongs to the closure $\overline{\mathfrak{D}}(\mathscr{K})$ of $\mathfrak{D}(\mathscr{K})$.

Clearly, the statement of $(1.5)$ carries over, and $\overline{\mathfrak{D}}(\mathscr{K})$ is again stable with respect to complementation.

A characterization of almost $\mathscr{K}$-determined sets requires a perturbation:

(4.5) Lemma. Let $A \in \mathfrak{A}$ and $f_{n} \in \mathscr{K}$ satisfy

$$
\left\{f_{n}>0\right\} \underset{0}{\rightarrow} A \text { and }\left\{f_{n}<0\right\} \underset{0}{\rightarrow} X \backslash A .
$$

If $\mathscr{K}$ is closed, there exist $f_{n}^{\prime} \in \mathscr{K}$ such that again

$$
\left\{f_{n}^{\prime}>0\right\} \underset{0}{\rightarrow} A \text { and }\left\{f_{n}^{\prime}<0\right\} \underset{0}{\rightarrow} X \backslash A
$$

and in addition

$$
\mu\left(f_{n}^{\prime}=0\right)=0 \text { for } n \in \mathbb{N} .
$$

Proof. The assumption implies

$$
\left\{f_{n}=0\right\}=\left\{f_{n} \leq 0\right\} \cap\left\{f_{n} \geq 0\right\} \underset{0}{\rightarrow}(X \backslash A) \cap A=\varnothing,
$$

hence $\operatorname{supp} \mathscr{K}=X$, and (1.10) provides $f_{0} \in \mathscr{K}$ such that $\left\{f_{0} \neq 0\right\} \underset{\mu}{=} X$. For fixed $n$, therefore, the sets $\left\{f_{n}+\varepsilon f_{0}=0\right\}, \varepsilon \in \mathbb{R}$, are pairwise disjoint modulo $\mu$. Now this measure is $\sigma$-finite, hence there is a sequence $\varepsilon_{n}^{m} \rightarrow 0$ such that

$$
\mu\left(f_{n}+\varepsilon_{n}^{m} f_{0}=0\right)=0 \text { for } m \in \mathbb{N} .
$$


Since the topology is metrizable, due to the convergence

$$
\left\{f_{n}+\varepsilon_{n}^{m} f_{0}>0\right\} \cap\left\{f_{n} \neq 0\right\} \underset{0}{\rightarrow}\left\{f_{n}>0\right\}
$$

with its analogue for $\left\{f_{n}<0\right\}$, there are coefficients $\varepsilon_{n}$ such that

$$
\left\{f_{n}^{\prime}>0\right\} \cap\left\{f_{n} \neq 0\right\} \underset{0}{\rightarrow} A \text { and }\left\{f_{n}^{\prime}<0\right\} \cap\left\{f_{n} \neq 0\right\} \underset{0}{\rightarrow} X \backslash A
$$

holds for $f_{n}^{\prime}=f_{n}+\varepsilon_{n} f_{0} \in \mathscr{K}$ and these functions satisfy in addition

$$
\mu\left(f_{n}^{\prime}=0\right)=0 \text { for } n \in \mathbb{N} .
$$

The assertion follows by another application of $\left\{f_{n}=0\right\} \underset{0}{\rightarrow} \varnothing$.

Now a first criterion for almost $\mathscr{K}$-determined sets can be derived:

(4.6) Theorem. Let $e \in \mathscr{L}_{0}(\mu)$ be arbitrary. Then a set $A \in \mathfrak{A}$ belongs to $\overline{\mathfrak{D}}(\mathscr{K})$ if and only if there exist functions $f_{n} \in \mathscr{K}$ such that

$$
\left\{f_{n}>e\right\} \underset{0}{\rightarrow} A \text { and }\left\{f_{n}<-e\right\} \underset{0}{\rightarrow} X \backslash A .
$$

Proof. 1. Assume first $A \in \overline{\mathscr{D}}(\mathscr{K})$ and fix a strictly positive function $h \in$ $\mathscr{L}_{1}(\mu)$. Then, by definition, there are sets $A_{n} \in \mathfrak{D}(\mathscr{K})$ such that

$$
\int_{X}\left|1_{A_{n}}-1_{A}\right| \wedge h d \mu \rightarrow 0
$$

and, according to (2.1), functions $f_{n} \in \mathscr{K}$ such that

$$
\int_{A_{n}}\left(h-f_{n}\right)^{+} d \mu+\int_{X \backslash A_{n}}\left(h+f_{n}\right)^{+} d \mu \rightarrow 0 .
$$

Due to the inequality

$$
\int_{X}\left|1_{\left\{f_{n}>0\right\}}-1_{A_{n}}\right| \wedge h d \mu \leq \int_{A_{n}}\left(h-f_{n}\right)^{+} d \mu+\int_{X \backslash A_{n}}\left(h+f_{n}\right)^{+} d \mu
$$

and its analogue for $\left\{f_{n}<0\right\}$ this combines to

$$
\left\{f_{n}>0\right\} \underset{0}{\overrightarrow{0}} A \text { and }\left\{f_{n}<0\right\} \underset{0}{\overrightarrow{0}} X \backslash A
$$

and settles the case $e=0$. Otherwise, for fixed $n$ and $m \rightarrow \infty$ obviously

$$
\left.\left\{f_{n}>\frac{|e|}{m}\right\} \rightarrow_{0}\left\{f_{n}>0\right\} \text { and }\left\{f_{n}<-\frac{|e|}{m}\right\} \rightarrow f_{0}<0\right\} .
$$

Since the topology is metrizable, this yields

$$
\left\{f_{n}^{\prime}>|e|\right\} \underset{0}{\rightarrow} A \text { and }\left\{f_{n}^{\prime}<-|e|\right\} \underset{0}{\rightarrow} X \backslash A
$$

for suitable multiples $f_{n}^{\prime}=m_{n} f_{n} \in \mathscr{K}$. But in view of

$$
\left\{f_{n}^{\prime}>|e|\right\} \subset\left\{f_{n}^{\prime}>e\right\} \subset\left\{f_{n}^{\prime} \geq-|e|\right\},
$$

with the lower and the upper bound both tending to $A$, this implies

$$
\left\{f_{n}^{\prime}>e\right\} \underset{0}{\rightarrow} A \text { and }\left\{f_{n}^{\prime}<-e\right\} \underset{0}{\vec{\longrightarrow}} X \backslash A \text { (similarly) }
$$

2. Conversely, let be given functions $f_{n} \in \mathscr{K}$ with this property. Then the inequalities

$$
\left\{f_{n}>e\right\} \cap\left\{f_{n} \geq-e\right\} \subset\left\{f_{n}>0\right\} \subset\left\{f_{n}>e\right\} \cup\left\{f_{n} \geq-e\right\}
$$


yield as above

$$
\left\{f_{n}>0\right\} \underset{0}{\rightarrow} A \text { and }\left\{f_{n}<0\right\} \underset{0}{\rightarrow} X \backslash A \text { (similarly), }
$$

i.e. it suffices to settle the case $e=0$. But in addition $\mathscr{K}$ may be assumed to be closed, hence the functions $f_{n}$ can be modified according to (4.5), which yields

$$
A_{n}=\left\{f_{n}^{\prime}>0\right\} \underset{0}{\rightarrow} A \text { and } A_{n} \in \mathfrak{S}(\mathscr{K}) .
$$

A reference to (1.7) completes the proof.

This theorem allows an explicit characterization of the class $\overline{\mathfrak{D}}(\mathscr{K})$, which uses the following notation:

(4.7) Definition. $\mathscr{K}^{* *}$ is the class of all functions $f^{* *} \in \widetilde{\mathscr{L}}_{0}(\mu)$ such that $f_{n} \rightarrow f^{* *}$ for some sequence $f_{n} \in \mathscr{K}, n \in \mathbb{N}$.

Here, the convergence modulo $\mu$ clearly can be replaced by convergence in measure. As in the case of $\mathscr{K}^{*}$ the class $\mathscr{K}^{* *}$ is stable under scalar multiplication. Moreover, passing from $\mathscr{K}$ to $\overline{\mathscr{K}}$ makes no difference, since for functions $f_{n}, f_{n}^{\prime} \in \mathscr{L}_{1}(\mu)$ certainly

$$
f_{n} \rightarrow f^{* *} \text { and } \sum_{n \in \mathbb{N}}\left\|f_{n}-f_{n}^{\prime}\right\|<\infty \text { implies } f_{n}^{\prime} \rightarrow f^{* *} .
$$

Now the statement of $(2.5)$ has the following counterpart:

(4.8) Theorem. A set $A \in \mathfrak{A}$ belongs to $\overline{\mathfrak{D}}(\mathscr{K})$ if and only if

$$
A=\left\{f^{* *}>0\right\} \quad \text { and } \quad X \backslash A=\underset{\mu}{=}\left\{f^{* *}<0\right\}
$$

for some function $f^{* *} \in \mathscr{K}^{* *}$.

Proof. 1. If $A \in \overline{\mathfrak{D}}(\mathscr{K})$, according to (4.6) there are $f_{n} \in \mathscr{K}$ such that

$$
\left\{f_{n}>1\right\} \underset{0}{\rightarrow} A \text { and }\left\{f_{n}<-1\right\} \underset{0}{\rightarrow} X \backslash A .
$$

By taking an appropriate subsequence this convergence can be interpreted as modulo $\mu$ as well, hence $\mathscr{K} \ni f_{1}+\cdots+f_{n} \rightarrow f^{* *}$, where

$$
f^{* *}=+\infty \text { on } A \text { and } f^{* *}=-\infty \text { on } X \backslash A .
$$

2. If, conversely, $A$ can be represented by means of $f^{* *}$ and $\mathscr{K} \ni f_{n} \rightarrow f^{* *}$, then

$$
\left\{f_{n}>0\right\} \underset{0}{\vec{\longrightarrow}}\left\{f^{* *}>0\right\}=A \text { and }\left\{f_{n}<0\right\} \underset{0}{\rightarrow}\left\{f^{* *}<0\right\}=X \backslash A,
$$

and the assertion is an immediate consequence of (4.6).

All comments concerning (2.5) carry over to (4.8): (1) $f^{* *} \in \mathscr{K}^{* *}$ actually can be restricted to the values $\pm \infty,(2)$ it does not suffice to consider only finitevalued functions $f^{* *},(3) \mathscr{K}^{* *}$ cannot be replaced by the limits of monotone sequences in $\mathscr{K}$. Again, (1) is contained in part 1 of the proof, while (2) and (3) follow from (3.3) for $\gamma=2$. Moreover, $A \in \overline{\mathfrak{D}}(\mathscr{K})$ is already a consequence of representations

$$
A=\underset{\mu}{=}\left\{f_{1}^{* *}>0\right\} \quad \text { and } \quad X \backslash A=\underset{\mu}{\bar{\mu}}\left\{f_{2}^{* *}<0\right\}
$$


with arbitrary $f_{i}^{* *} \in \mathscr{K}^{* *}$ as follows in analogy to the corresponding statement concerning (2.5).

In addition, it should be mentioned that, in contrast to $\mathfrak{D}(\mathscr{K}) \subset \overline{\mathfrak{D}}(\mathscr{K})$, the inclusion $\mathscr{K}^{*} \subset \mathscr{K}^{* *}$, though valid for countable spaces $X$, may fail in more general situations.

To conclude this section, the approximation problem will be settled:

(4.9) Theorem. If $\mathscr{K}$ is closed, then $\mathfrak{S}(\mathscr{K})$ is dense in $\mathfrak{D}(\mathscr{K})$.

Proof. For an arbitrary set $A \in \mathfrak{D}(\mathscr{K})$ according to (4.6) there exist functions $f_{n} \in \mathscr{K}$ such that

$$
\left\{f_{n}>0\right\} \underset{0}{\vec{\rightarrow}} A \text { and }\left\{f_{n}<0\right\} \underset{0}{\rightarrow} X \backslash A .
$$

Since $\mathscr{K}$ is closed, (4.5) applies and yields $\mathfrak{S}(\mathscr{K}) \ni\left\{f_{n}^{\prime}>0\right\} \underset{0}{\rightarrow} A$.

As is clear from (1.8), the condition $\overline{\mathscr{K}}=\mathscr{K}$ cannot be dispensed with. Moreover, as a consequence of this theorem and the various counterexamples it follows that in the chain

$$
\mathfrak{S}(\mathscr{K}) \subset \cdots \subset \mathfrak{S}_{\gamma}(\mathscr{K}) \subset \cdots \subset \mathfrak{D}(\mathscr{K}) \subset \overline{\mathfrak{D}}(\mathscr{K})
$$

only the last member is necessarily closed.

All classes in this chain coincide in a simple special case:

(4.10) Corollary. If $X$ is finite, then $\mathfrak{S}(\mathscr{K})=\overline{\mathfrak{D}}(\mathscr{K})$.

Proof. $\mathscr{K}$ is finite dimensional, hence closed, and thus (4.9) is applicable. Therefore each set $A \in \overline{\mathfrak{D}}(\mathscr{K})$ is the limit of a sequence in $\mathfrak{S}(\mathscr{K})$, which is eventually constant, because $X$ is finite.

\section{Weak UNIQUeness}

In some applications it is more adequate to work with the following weaker notion of uniqueness:

(5.1) Definition. A set $A \in \mathfrak{A}$ is called "weakly $\mathscr{K}$-determined", if

$$
A \underset{\mathscr{K}}{\widetilde{A}} B \in \mathfrak{A} \text { implies } B \underset{\mu}{=} A .
$$

This condition is clearly satisfied for $A \in \mathfrak{D}(\mathscr{K})$, but the converse fails:

(5.2) Example. Let $X$ be a nonempty subset of $\mathbb{N}$,

$$
\mu(\{n\})>\sum_{n<m \in X} \mu(\{m\}) \text { for } n \in X,
$$

and $\mathscr{K}$ consist of all constant functions. Then each subset of $X$ turns out to be weakly $\mathscr{K}$-determined, while it was observed in (1.3) that $\mathfrak{D}(\mathscr{K})$ contains only $\varnothing$ and $X$.

Since convexity is lost by the passage from $\mathscr{G}$ to $\mathfrak{A}$, the notion in $(5.1)$ is less convenient to work with. There is, however, one important exception, present under a condition first considered in [10] and in a way complementary to (1.13): 
(5.3) Definition. $\mathscr{K}$ is called "thin", if the equivalence $1_{C} g \widetilde{\mathscr{H}}_{0} 0$ for each $C \in \mathfrak{A}$ with $\mu(C)>0$ has a nontrivial solution $g \in \mathscr{L}_{\infty}(\mu)$, i.e. a solution satisfying $\mu(\{g \neq 0\} \cap C)>0$.

To see this condition to be natural, observe that for a set $C$ not meeting the requirement functions $g$ vanishing outside of $C$ can be identified by the integrals $\int_{X} f g d \mu, f \in \mathscr{K}$. Clearly, for being thin the passage from $\mathscr{K}$ to $\overline{\mathscr{K}}$ makes no difference. Moreover, provided supp $\mathscr{K}=\underset{\mu}{=} X$, the underlying measure $\mu$ has to be nonatomic. In this case the well-known convexity theorem of Liapunov [13] implies that each finite-dimensional subspace $\mathscr{K}$ of $\mathscr{L}_{1}(\mu)$ is thin (see e.g. [6, p. 106]).

The crucial property of thin subspaces $\mathscr{K}$ in this context consists in the following fact (see [10] for finite $\mu$ and [21] for $\sigma$-finite $\mu$ ):

$$
\left\{\begin{array}{l}
\text { for each } g \in \mathscr{G} \text { there exists } A \in \mathfrak{A} \text { such that } \\
A \subset\{g>0\} \text { and } 1_{A} \tilde{\mathscr{K}} g .
\end{array}\right.
$$

This implies in particular that only indicator functions have a chance to be determined by the integrals $\int_{X} f g d \mu, f \in \mathscr{K}$.

The main consequence of $(*)$ is the coincidence of weak and strong uniqueness:

(5.4) Theorem. If $\mathscr{K}$ is thin, then each weakly $\mathscr{K}$-determined set $A$ is contained in $\mathfrak{D}(\mathscr{K})$.

Proof. Consider an equivalence $1_{A} \tilde{\mathscr{K}} g \in \mathscr{G}$ and choose an arbitrary subset $B \in \mathfrak{A}$ of $X \backslash A$. Then by property $(*)$ there exist sets $B^{\prime}, B^{\prime \prime} \in \mathfrak{A}$ satisfying

$$
B^{\prime} \subset B \quad \text { and } \quad 1_{B^{\prime}} \tilde{\mathscr{K}}_{B} 1_{B}, \quad B^{\prime \prime} \subset X \backslash B \text { and } 1_{B^{\prime \prime}} \tilde{\mathscr{K}}_{X \backslash B} g .
$$

Therefore, $B^{\prime}$ and $B^{\prime \prime}$ being disjoint,

$$
1_{A} \widetilde{\mathscr{K}}_{B} 1_{B} g+1_{X \backslash B} g \underset{\mathscr{K}}{\sim} 1_{B^{\prime}}+1_{B^{\prime \prime}}=1_{B^{\prime} \cup B^{\prime \prime}}
$$

and thus, $A$ being weakly $\mathscr{K}$-determined, $A \underset{\mu}{=} B^{\prime} \cup B^{\prime \prime}$. But this implies in particular $B^{\prime}=\varnothing$, hence

$$
1_{B} g \underset{\mathscr{K}}{\sim} 1_{B^{\prime}}=0 \text { for all } B \in \mathfrak{A} \text { with } B \subset X \backslash A
$$

or equivalently

$$
1_{C}\left(1_{X \backslash A} g\right) \underset{\mathscr{K}}{\sim} 0 \text { for all } C \in \mathfrak{A} .
$$

Since $A$ is weakly $\mathscr{K}$-determined, the condition supp $\mathscr{K}=\overline{\bar{\mu}} X$ is certainly satisfied, and (1.13) applies with the result $1_{X \backslash A} g \underset{\mu}{=} 0$. Now it follows as in (1.5) that $X \backslash A$ is weakly $\mathscr{K}$-determined as well. Since $1_{X \backslash A} \widetilde{\mathscr{K}} 1-g$, therefore similarly $1_{A}(1-g) \underset{\mu}{=} 0$. When combined, the last two equations yield $g \underset{\mu}{=}{ }_{A}$, proving $A \in \mathfrak{D}(\mathscr{K})$.

\section{Classical case}

The most important application of (5.4) concerns the geometric situation as outlined in the introduction in the context of computer tomography. To ensure 
the existence of nontrivial integrable functions of type $f_{i} \circ \pi_{i}$, in this section the basic space will be assumed to be bounded, hence without loss of generality

$$
\left.X=\prod_{1 \leq i \leq n} X_{i} \quad \text { with } X_{i}=\right] 0,1[\text { and } n>1 .
$$

Endow $X$ with its Borel $\sigma$-algebra $\mathfrak{A}$ and the normed measure

$$
\mu=\bigotimes_{1 \leq i \leq n} \mu_{i} \text { with } \mu_{i}=\lambda
$$

where $\lambda$ denotes Lebesgue measure. In $\mathscr{L}_{1}(\mu)$ consider the linear space

$$
\mathscr{K}=\left\{\sum_{1 \leq i \leq n} f_{i} \circ \pi_{i}: f_{i} \in \mathscr{L}_{1}\left(\mu_{i}\right)\right\} .
$$

In view of (4.9) the following simple result, contrasting with the discrete situation in (4.2), is of interest:

(6.1) Proposition. $\mathscr{K}$ is a closed subspace of $\mathscr{L}_{1}(\mu)$.

Proof. Defining $X_{\bar{i}}=\prod_{j \neq i} X_{j}$ and $\mu_{\bar{i}}=\bigotimes_{j \neq i} \mu_{j}$ introduce linear maps $\varphi_{i}$ from $\mathscr{L}_{1}(\mu)$ into $\mathscr{L}_{1}\left(\mu_{i}\right)$ by

$$
\varphi_{i}(f): x_{i} \mapsto \int_{X_{\bar{i}}} f_{x_{i}} d \mu_{\bar{i}},
$$

where by Fubini's theorem

$$
\left\|\varphi_{i}(f) \circ \pi_{i}\right\| \leq\|f\| \text { for } 1 \leq i \leq n .
$$

Let now $f^{k}=\sum_{1 \leq i \leq n} f_{i}^{k} \circ \pi_{i}$ with $f_{i}^{k} \in \mathscr{L}_{1}\left(\mu_{i}\right)$ satisfy

$$
\left\|f^{k}-f\right\| \rightarrow 0 \text { for some } f \in \mathscr{L}_{1}(\mu) \text {. }
$$

Then, in particular,

$$
\sum_{1 \leq i \leq n} \int_{X_{i}} f_{i}^{k} d \mu \rightarrow \int_{X} f d \mu
$$

hence, adding appropriate constants, without loss of generality

$$
\int_{X_{i}} f_{i}^{k} d \mu_{i}=0 \text { and } \int_{X} f d \mu=0 .
$$

This implies

$$
f^{k}=\sum_{1 \leq i \leq n} \varphi_{i}\left(f^{k}\right) \circ \pi_{i},
$$

hence $f$ may be replaced by

$$
f^{\prime}=\sum_{1 \leq i \leq n} \varphi_{i}(f) \circ \pi_{i} \in \mathscr{K},
$$

because, according to the first part of the proof,

$$
\left\|f^{k}-f^{\prime}\right\|=\left\|\sum_{1 \leq i \leq n} \varphi_{i}\left(f^{k}-f\right) \circ \pi_{i}\right\| \leq \sum_{1 \leq i \leq n}\left\|f^{k}-f\right\| \rightarrow 0 \text { for } k \rightarrow \infty .
$$


The subspace $\mathscr{K}$ is not only closed but also thin, as follows from more general results obtained independently in [7] and [17]. It makes no difference, therefore, to study (strongly) $\mathscr{K}$-determined sets or weakly $\mathscr{K}$-determined sets.

In this context it should be mentioned that $\mathscr{K}$ remains thin, if the canonical projections $\pi_{i}$ are replaced by any finite number of linear maps $\varphi_{i}$ as considered in the introduction (for a formal proof see [16]). This allows to settle two questions touched there by an application of the crucial property $(*)$ stated after (5.3). First, without any restriction by some upper bound, for every nontrivial nonnegative function $g \in \mathscr{L}_{\infty}(\mu)$ there exists a nonnegative function $g^{\prime} \in \mathscr{L}_{\infty}(\mu)$ such that $g^{\prime} \underset{\mathscr{K}}{\sim} g$ without $g^{\prime}=g$. Indeed, this is immediate unless $g$ is itself an indicator function $1_{B}$; in this case, however, it suffices to choose $A \in \mathfrak{A}$ with $1_{A} \underset{\mathscr{K}}{\sim} \frac{1}{2} 1_{B}$ and to consider $g^{\prime}=2 \cdot 1_{A}$. Moreover, as pointed out already in $\S 5$, if the upper bound 1 is present, only indicator functions can be uniquely determined by their equivalence class.

After this digression the study of the classical case will be continued. Here the result is very satisfactory in dimension 2: employing profound results by Lorentz [15], in the present notation, Kuba and Volcic [12] and Fishburn et al. [3] proved the equation $\mathfrak{S}(\mathscr{K})=\mathfrak{D}(\mathscr{K})$. More generally the following result holds:

(6.2) Proposition. In the case $n=2$ the class $\mathfrak{S}(\mathscr{K})$ is closed, i.e.

$$
\mathfrak{S}(\mathscr{K})=\overline{\mathfrak{D}}(\mathscr{K}) \text {. }
$$

Proof. 1. It remains only to prove that $\mathfrak{D}(\mathscr{K})$ is closed. To this end the uniqueness criterion in [15] will be needed explicitly. Taking up the notation in the proof of $(6.1)$, assign a pair of "cross functions"

$$
\varphi_{i}^{A}=\varphi_{i}\left(1_{A}\right): x_{i} \mapsto \mu_{\bar{i}}\left(A_{x_{i}}\right)
$$

to $A \in \mathfrak{A}$ and let $\psi_{i}^{A}$ be the associated (upper) distribution function

$$
\psi_{i}^{A}(t)=\mu_{i}\left(\varphi_{i}^{A} \geq t\right) \text { for } 0 \leq t \leq 1 .
$$

With these notations $A$ is $\mathscr{K}$-determined if and only if

$$
\int_{0}^{t} \psi_{1}^{A}(s) d s=\int_{0}^{1}\left(\psi_{2}^{A}(s) \wedge t\right) d s \quad \text { for } 0 \leq t \leq 1
$$

(or equivalently with the indices interchanged).

2. Consider now a sequence of sets $A_{n} \in \mathfrak{D}(\mathscr{K})$ with $A_{n} \overrightarrow{0} A$ and denote by $\varphi_{i n}, \varphi_{i}$ and $\psi_{i n}, \psi_{i}$ the functions corresponding to $A_{n}, A$. Since $\mu$ is finite, convergence in measure implies norm convergence and, by the contraction inequality in the proof of $(6.1)$, the cross functions $\varphi_{i n}$ converge to $\varphi_{i}$ in the norm as well. But this implies convergence of the distribution functions $\psi_{\text {in }}$ to $\psi_{i}$ at all continuity points of the limit. This, finally, yields the convergence of the integrals in the criterion $(*)$ and proves $A \in \mathfrak{D}(\mathscr{K})$.

In the case $n>2$ the situation is much more involved. That $\mathfrak{D}(\mathscr{K})$ is no longer closed, has been shown by Fishburn et al. [3]; that the class $\mathfrak{S}_{\gamma}(\mathscr{K})$ depends on $\gamma$ at least at the beginning, i.e. $\mathfrak{S}_{1}(\mathscr{K}) \neq \mathfrak{S}_{2}(\mathscr{K})$, is contained in a counterexample due to Kemperman [9]. Therefore the main open problem is to decide whether $\mathfrak{D}(\mathscr{K})$ is exhausted by the classes $\mathfrak{S}_{\gamma}(\mathscr{K})$. 
To show that this fails, too, requires a slight generalization of a result mentioned without proof in [9]:

(6.3) Lemma. Assume $n \geq 2$ and let $\nu_{1}, \ldots, \nu_{n}$ be $\sigma$-finite measures on $\mathbb{R}$ with full support and with product measure $\nu$ on $\mathbb{R}^{n}$. Then for functions $e_{i} \in$ $\mathscr{L}_{0}\left(\nu_{i}\right)$ satisfying

$$
\begin{aligned}
& S_{-}=\left\{y \in \mathbb{R}^{n}: \sum_{1 \leq i \leq n} y_{i}<0\right\} \underset{\nu}{\subset}\left\{y \in \mathbb{R}^{n}: \sum_{1 \leq i \leq n} e_{i}\left(y_{i}\right) \leq 0\right\}, \\
& S_{+}=\left\{y \in \mathbb{R}^{n}: \sum_{1 \leq i \leq n} y_{i}>0\right\} \underset{\nu}{\subset}\left\{y \in \mathbb{R}^{n}: \sum_{1 \leq i \leq n} e_{i}\left(y_{i}\right) \geq 0\right\}
\end{aligned}
$$

there exist constants $a \geq 0$ and $b_{i}$ with $\sum_{1 \leq i \leq n} b_{i}=0$ such that

$$
e_{i}\left(y_{i}\right)=a y_{i}+b_{i} \text { for } \nu_{i} \text {-almost all } y_{i}, 1 \leq i \leq n .
$$

Proof. 1. With respect to $\nu_{i}=\bigotimes_{j \neq i} \nu_{j}$ define the functions

$$
\begin{aligned}
& \underline{e}_{i}\left(y_{i}\right)=\nu_{i}-\sup \left\{-\sum_{j \neq i} e_{j}\left(y_{j}\right): \sum_{j \neq i} y_{j}>-y_{i}\right\}, \\
& \bar{e}_{i}\left(y_{i}\right)=\nu_{\bar{i}}-\inf \left\{-\sum_{j \neq i} e_{j}\left(y_{j}\right): \sum_{j \neq i} y_{j}<-y_{i}\right\} .
\end{aligned}
$$

Since the sets defined by $\sum_{j \neq i} y_{j}>-y_{i}$ resp. $\sum_{j \neq i} y_{j}<-y_{i}$ for each $y_{i}$ have positive $\nu_{i}$-measure, these functions are everywhere finite-valued. The following properties are immediate:

$\underline{e}_{i}$ and $\bar{e}_{i}$ are nondecreasing,

$\underline{e}_{i}$ is left continuous and $\bar{e}_{i}$ is right continuous .

Moreover, due to the assumption on $S_{-}$and $S_{+}$, by Fubini's theorem

$$
\begin{aligned}
& \underline{e}_{i} \leq e_{i} \leq \bar{e}_{\nu_{i}}, \\
& \underline{e}_{i}\left(y_{i}\right) \leq \bar{e}_{i}\left(y_{i}\right) \text { for all } y_{i},
\end{aligned}
$$

where (4) is a consequence of (1)-(3) and the fact that $\nu_{i}$ has full support. For the same reason

$$
\bar{e}_{i}\left(y_{i}\right) \leq \underline{e}_{i}\left(z_{i}\right) \text { for } y_{i}<z_{i} .
$$

2. Now the crucial point is the relation

$$
\underline{e}_{i}\left(y_{i}\right)+\sum_{j \neq i} \bar{e}_{j}\left(y_{j}\right) \leq 0 \leq \bar{e}_{i}\left(y_{i}\right)+\sum_{j \neq i} \underline{e}_{j}\left(y_{j}\right) \text { for } \sum_{1 \leq k \leq n} y_{k}=0 .
$$

Indeed, choose $x_{1}, \ldots, x_{n}$ such that

$$
x_{i}<y_{i}, x_{j}>y_{j} \text { for } j \neq i \text { and } \sum_{1 \leq k \leq n} x_{k} \leq 0
$$

and consider for arbitrary $\varepsilon>0$ the set

$$
Z=\left\{z \in \mathbb{R}^{n}: x_{i}-\varepsilon<z_{i}<x_{i} \text { and } y_{j}<z_{j}<x_{j} \text { for } j \neq i\right\},
$$


which obviously is contained in $S_{-}$and has positive measure. Thus

hence by (3)

$$
\sum_{1 \leq i \leq n} e_{i}\left(z_{i}\right) \leq 0 \text { for } \nu \text {-almost all } z \in Z,
$$

$$
\sum_{1 \leq i \leq n} \underline{e}_{i}\left(z_{i}\right) \leq 0 \text { for } \nu \text {-almost all } z \in Z .
$$

This, due to (5), implies

$$
\left.\underline{e}_{i}\left(z_{i}\right)+\sum_{j \neq i} \bar{e}_{j}\left(y_{j}\right) \leq 0 \text { for } \nu_{i} \text {-almost all } z_{i} \in\right] x_{i}-\varepsilon, x_{i}[
$$

and, due to (1), leads to

$$
\underline{e}_{i}\left(x_{i}-\varepsilon\right)+\sum_{j \neq i} \bar{e}_{j}\left(y_{j}\right) \leq 0 .
$$

For $x_{i} \uparrow y_{i}$ and $\varepsilon \downarrow 0$ this yields by (2) the first half of the assertion, while the second half follows by symmetry.

3. Summing up the inequalities (6) over $i$ yields

$$
\sum_{1 \leq i \leq n} \underline{e}_{i}\left(y_{i}\right)+(n-1) \sum_{1 \leq i \leq n} \bar{e}_{i}\left(y_{i}\right) \leq \sum_{1 \leq i \leq n} \bar{e}_{i}\left(y_{i}\right)+(n-1) \sum_{1 \leq i \leq n} \underline{e}_{i}\left(y_{i}\right)
$$

whenever $\sum_{1 \leq i \leq n} y_{i}=0$, and in view of $n>2$ this is equivalent to

$$
\sum_{1 \leq i \leq n} \bar{e}_{i}\left(y_{i}\right) \leq \sum_{1 \leq i \leq n} \underline{e}_{i}\left(y_{i}\right) \text { for } \sum_{1 \leq i \leq n} y_{i}=0
$$

By suitable choice of $y_{i}, j \neq i$, this combines with (4) to

$$
\underline{e}_{i}\left(y_{i}\right)=\bar{e}_{i}\left(y_{i}\right) \text { for all } y_{i} \text {. }
$$

Therefore, by (3)

$$
\underline{e}_{i} \underset{\nu_{i}}{=} e_{i} \underset{\nu_{i}}{=} \bar{e}_{i}
$$

and by (6)

$$
\sum_{1 \leq i \leq n} \underline{e}_{i}\left(y_{i}\right)=0=\sum_{1 \leq i \leq n} \bar{e}_{i}\left(y_{i}\right) \text { for } \sum_{1 \leq i \leq n} y_{i}=0 .
$$

4. In view of (1), (7), and (8) it remains to find the nondecreasing solutions $e_{1}, \ldots, e_{n}$ of the functional equation

$$
\sum_{1 \leq i \leq n} e_{i}\left(y_{i}\right)=0 \text { for } \sum_{1 \leq i \leq n} y_{i}=0
$$

Here, the values $e_{i}(0)$ correspond to the constants $b_{i}$, hence $e_{i}(0)=0$ can be assumed. For $i \neq j$-and suitable $y_{k}, k \neq i, j$-equation (9) yields

$$
e_{i}(y)=e_{i}(y)+e_{j}(0)=e_{i}(0)+e_{j}(y)=e_{j}(y),
$$

hence $e$ may be written instead of $e_{i}$. Once more using (9) and $n>2$ leads to the functional equation

$$
e(y+z)=e(y+z)+e(0)=e(y)+e(z),
$$

and the assertion follows from the isotony of $e$. 
It should be noted that the condition $n>2$ is essential for this result, because for $n=2$ each nondecreasing function $e$ provides the solution

$$
e_{1}\left(y_{1}\right)=e\left(y_{1}\right) \text { and } e_{2}\left(y_{2}\right)=-e\left(-y_{2}\right) .
$$

Now the announced counterexample can be given:

(6.4) Proposition. Assume $n>2$ and let $\varphi_{i}: X_{i} \rightarrow \mathbb{R}$ be homeomorphisms satisfying

$$
\begin{gathered}
\int_{X_{i}} \varphi_{i}^{-} d \mu_{i}<\infty \text { for all } i, \\
\int_{X_{i}} \varphi_{i}^{+} d \mu_{i}=\infty \text { for at least one } i .
\end{gathered}
$$

Then the set

$$
A=\left\{\sum_{1 \leq i \leq n} \varphi_{i} \circ \pi_{i}>0\right\}
$$

is $\mathscr{K}$-determined without being $\mathscr{K}$-separated of any order $\gamma$.

Proof. 1. The functions

$$
\sum_{1 \leq i \leq n}\left(\varphi_{i} \wedge k\right) \circ \pi_{i} \in \mathscr{K}
$$

increase for $k \rightarrow \infty$ to the function

$$
f^{*}=\sum_{1 \leq i \leq n} \varphi_{i} \circ \pi_{i} \in \mathscr{K}^{*} .
$$

Since $\mu\left(f^{*}=0\right)=0$, the assertion $A \in \mathfrak{D}(\mathscr{K})$ follows from (2.5).

2. Now assume for some countable ordinal $\gamma>0$ a representation

$$
A \underset{\mu}{=}\left\{\left(f^{\alpha}, \alpha<\gamma\right) \succ 0\right\} \quad \text { with } f^{\alpha}=\sum_{1 \leq i \leq n} f_{i}^{\alpha} \circ \pi_{i} \in \mathscr{K},
$$

where $\left\|f^{0}\right\| \neq 0$ without loss of generality. This implies in particular

$$
\left\{\sum_{1 \leq i \leq n} f_{i}^{0} \circ \pi_{i}>0\right\} \underset{\mu}{\subset} A \underset{\mu}{\subset}\left\{\sum_{1 \leq i \leq n} f_{i}^{0} \circ \pi_{i} \geq 0\right\} .
$$

Passing from $X$ to $\mathbb{R}^{n}$ via $y_{i}=\varphi_{i}\left(x_{i}\right), 1 \leq i \leq n$, yields

$$
\left\{y \in \mathbb{R}^{n}: \sum_{1 \leq i \leq n} y_{i}<0\right\} \subset \subset_{\nu}\left\{y \in \mathbb{R}^{n}: \sum_{1 \leq i \leq n} f_{i}^{0}\left(\varphi_{i}^{-1}\left(y_{i}\right)\right) \leq 0\right\}
$$

and its counterpart, where $\nu$ is the product of the image measures $\nu_{i}$ of $\mu_{i}$ under $\varphi_{i}$. Since the measures $\nu_{i}$ have full support, (6.3) applies to $e_{i}=f_{i}^{0} \circ \varphi_{i}^{-1}$ and leads to

$$
\left(f_{i}^{0} \circ \varphi_{i}^{-1}\right)\left(y_{i}\right)=a y_{i}+b_{i} \quad \text { for } \nu_{i} \text {-almost all } y_{i} \in \mathbb{R}
$$


with $a \geq 0$ and $\sum_{1 \leq i \leq n} b_{i}=0$. Finally, returning from $\mathbb{R}^{n}$ to $X$, this yields

$$
f_{i}^{0} \underset{\mu_{i}}{=} a \varphi_{i}+b_{i} \text { for } 1 \leq i \leq n,
$$

where the case $a=0$ is excluded by $\left\|f^{0}\right\| \neq 0$. Therefore $\varphi_{i} \in \mathscr{L}_{1}(\mu)$ for all $i$, in contradiction to the hypothesis.

It should be noted that the set $A$ is by no means exotic; in fact it is always open and connected.

\section{REFERENCES}

1. R. Anantharaman and K. M. Garg, Some topological properties of vector measures and their integral maps, J. Austral. Math. Soc. (Ser. A) 23 (1977), 453-466.

2. R. Chen and L. A. Shepp, On the sum of random variables, Amer. Statist. 37 (1983), 237.

3. P. C. Fishburn, J. C. Lagarias, J. A. Reeds, and L. A. Shepp, Sets uniquely determined by projections on axes. I. Continuous case, SIAM J. Appl. Math. 50 (1990), 288-306.

4. __ Sets uniquely determined by projections on axes. II. Discrete case, Discrete Math. 91 (1991), 149-159.

5. R. J. Gardner, Measure theory and some problems in geometry, Atti Sem. Mat. Fis. Univ. Modena 39 (1991), 51-72.

6. R. Holmes, Geometric functional analysis and its applications, Springer-Verlag, Berlin, Heidelberg, and New York, 1975.

7. H. G. Kellerer, Schnittmaß-Funktionen in mehrfachen Produkträumen, Math. Ann. 155 (1964), 369-391.

8. J. H. B. Kemperman, On sets that are uniquely determined by a restricted set of integrals, Trans. Amer. Math. Soc. 322 (1990), 417-458.

9. __ Sets of uniqueness and systems of inequalities having a unique solution, Pacific $\mathbf{J}$. Math. 148 (1991), 275-301.

10. J. Kingman and A. Robertson, On a theorem of Liapunov, J. London Math. Soc. 43 (1968), 347-351.

11. I. Kluvánek and G. Knowles, Vector measures and control systems, North-Holland, Amsterdam and Oxford, 1975.

12. A. Kuba and A. Volcic, Characterization of measurable plane sets which are reconstructable from their two projections, Inverse Problems 4 (1988), 513-527.

13. A. Liapunov, Sur les fonctions-vecteurs complètement additives, Izv. Akad. Nauk SSSR Ser. Mat. 4 (1940), 465-478. (Russian)

14. J. Lindenstrauss, A remark on extreme doubly stochastic measures, Amer. Math. Monthly 72 (1965), 379-382.

15. G. G. Lorentz, A problem of plane measure, Amer. J. Math. 71 (1949), 417-426.

16. S. Marx, Schnittmaßprobleme im $R^{d}$ bezüglich endlich vieler Teilräume, Diploma thesis, Universität Erlangen, 1988.

17. I. V. Romanovskii and V. N. Sudakov, On the existence of independent partitions, Proc. Steklov Inst. Math. 79 (1965), 1-7.

18. G. Simons, An unexpected expectation, Ann. Probab. 5 (1977), 157-158.

19. A. Spakowski, Openness of vector measures and their integral maps, J. Austral. Math. Soc. (Ser. A) 45 (1988), 351-359.

20. V. N. Sudakov, Geometric problems in the theory of infinite-dimensional probability distributions, Proc. Steklov Inst. Math. 141 (1979), 1-178.

21. R. Wegmann, Der Wertebereich von Vektorintegralen, Z. Wahrsch. Verw. Gebiete 14 (1970), 203-238.

Mathematisches Institut, Universität München, Theresienstrasse 39, 8 MünChen 2 , GeRMANY

E-mail address: H.Kellerer@Mathematik.Uni-Muenchen.DBP.DE 